\title{
COHOMOLOGY OF COXETER ARRANGEMENTS AND SOLOMON'S DESCENT ALGEBRA
}

\section{J. MATTHEW DOUGLASS, GÖTZ PFEIFFER, AND GERHARD RÖHRLE}

Abstract. We refine a conjecture by Lehrer and Solomon on the structure of the Orlik-Solomon algebra of a finite Coxeter group $W$ and relate it to the descent algebra of $W$. As a result, we claim that both the group algebra of $W$ and the Orlik-Solomon algebra of $W$ can be decomposed into a sum of induced one-dimensional representations of element centralizers, one for each conjugacy class of elements of $W$. We give a uniform proof of the claim for symmetric groups. In addition, we prove that a relative version of the conjecture holds for every pair $\left(W, W_{L}\right)$, where $W$ is arbitrary and $W_{L}$ is a parabolic subgroup of $W$, all of whose irreducible factors are of type $A$.

\section{Contents}

1. Introduction

2. The Orlik-Solomon algebra and Solomon's descent algebra

3. BBHT idempotents

4. $E_{\lambda}$ is an induced representation

5. Symmetric groups: $\lambda=(n)$

6. Symmetric groups: Arbitrary $\lambda$

7. Parabolic subgroups of type $A$

\section{INTRODUCTION}

Suppose $V$ is a finite-dimensional, complex vector space. A linear transformation $t$ in $\operatorname{GL}(V)$ is called a reflection if it has finite order and the fixed point set of $t$ is a hyperplane in $V$, or equivalently, if $t$ is diagonalizable with eigenvalues 1 , with multiplicity $\operatorname{dim} V-1$, and $\zeta$, where $\zeta$ is a root of unity, with multiplicity 1 . Suppose that $W$ is a finite subgroup of $\mathrm{GL}(V)$ generated by a set of reflections $T$. For each $t$ in $T$, let $H_{t}=\operatorname{Fix}(t)$ denote the fixed point set of $t$ in $V$ and set $\mathcal{A}=\left\{H_{t} \mid t \in T\right\}$ and $M_{W}=V \backslash \bigcup_{t \in T} H_{t}$. Then $(V, \mathcal{A})$ is a hyperplane arrangement, and the complement $M_{W}$ is an open, $W$-stable subset of $V$.

The action of $W$ on $M_{W}$ determines a representation of $W$ on the singular cohomology of $M_{W}$. For $p \geq 0$ let $H^{p}\left(M_{W}\right)$ denote the $p^{\text {th }}$ singular cohomology space of $M_{W}$ with complex coefficients and let $H^{*}\left(M_{W}\right)=\bigoplus_{p>0} H^{p}\left(M_{W}\right)$ denote the total cohomology of $M_{W}$, a graded $\mathbb{C}$-vector space. It follows from a result

Received by the editors July 16, 2012 and, in revised form, December 4, 2012.

2010 Mathematics Subject Classification. Primary 20F55; Secondary 05E10, 52C35.

The authors would like to thank their charming wives for their unwavering support during the preparation of this paper. 
of Brieskorn [11] that $\operatorname{dim} H^{*}\left(M_{W}\right)=|W|$, and so a naive guess would be that the representation of $W$ on $H^{*}\left(M_{W}\right)$ is the regular representation of $W$. A simple computation for the symmetric group $S_{3}$ shows that this is not the case.

In 1987, Lehrer 25] determined the character of the representation of $W$ on $H^{*}\left(M_{W}\right)$ when $W=S_{n}$ is a symmetric group by explicitly computing the "equivariant Poincaré polynomials" $P_{S_{n}}(g, t)=\sum_{p \geq 0} \operatorname{trace}\left(g, H^{p}\left(M_{W}\right)\right) t^{p}$, for $g$ in $S_{n}$ (here, $t$ is an indeterminate). Subsequently, equivariant Poincaré polynomials were computed case by case for other groups by various authors. In 2001, Blair and Lehrer 9] showed that for any complex reflection group, the equivariant Poincaré polynomials have the form $P_{W}(g, t)=\sum_{x \in Z_{W}(g)} f_{g}(x)(-t)^{c(x)}$, where $f_{g}: Z_{W}(g) \rightarrow \mathbb{C}$ is explicitly given and $c(x)$ is the codimension of the fixed point space of $x$ in $V$. Felder and Veselov 14 have found an elegant description of the character of $H^{*}\left(M_{W}\right)$ when $W$ is a Coxeter group that precisely describes how the character of $H^{*}\left(M_{W}\right)$ differs from the regular character $\rho$ of $W$. Specifically, Felder and Veselov show that the character of $H^{*}\left(M_{W}\right)$ is given as

$$
\sum_{t}\left(2 \operatorname{Ind}_{\langle t\rangle}^{W}(1)-\rho\right)
$$

where the sum runs over a set of "special" involutions $t$ in $W$.

In contrast, while the representation of $W$ on $H^{*}\left(M_{W}\right)$ is well understood, much less is known about the representations of $W$ on the individual graded pieces $H^{p}\left(M_{W}\right)$ for $p \geq 0$. When $W$ is a symmetric group, Lehrer and Solomon [26] have described these representations as sums of representations induced from linear characters of centralizers of elements in $W$. They conjecture that a similar decomposition exists in general.

For the symmetric group $S_{n}$, Barcelo and Bergeron [1] construct an explicit $S_{n^{-}}$ stable subspace of the exterior algebra of the free Lie algebra on $n$ letters that affords the character of $H^{*}\left(M_{W}\right)$ tensored with the sign character. Their construction could be used to study the characters of the individual cohomology spaces $H^{p}\left(M_{W}\right)$.

For the hyperoctahedral group $W\left(B_{n}\right)$, Douglass [12] extended the construction of Lehrer and Solomon and expressed each $H^{p}\left(M_{W}\right)$ as a sum of representations induced from linear characters of subgroups. However, the subgroups appearing are not always centralizers of elements of $W\left(B_{n}\right)$. At the same time, Bergeron used the free Lie algebra on $2 n$ letters to construct a representation of $W\left(B_{n}\right)$ analogous to the representation of $S_{n}$ constructed in [1]. The character of this representation of $W\left(B_{n}\right)$ is again the character of $H^{*}\left(M_{W}\right)$ tensored with the sign character. He then uses this construction to study the characters of the individual cohomology spaces $H^{p}\left(M_{W}\right)$.

In this paper we state a conjecture for a finite Coxeter group $W$ (Conjecture 2.1) that both refines the conjecture of Lehrer and Solomon [26, Conjecture 1.6] and directly relates the representation of $W$ on $H^{p}\left(M_{W}\right)$ to a subrepresentation of the right regular representation of $W$. It is straightforward to see that Conjecture 2.1 holds for $W$ if and only if it holds for every irreducible factor of $W$. Thus, to prove the conjecture we may assume that $W$ is irreducible. Conjecture 2.1 is proved for symmetric groups in this paper (Theorem 6.3). The conjecture has been proven for all rank two Coxeter groups [13] and has been checked using the computer algebra system GAP [32] and the package CHEVIE [16] for all Coxeter groups with rank eight or less $([6], 7],[])$. More generally, in $\$ 7$ we extend the constructions used in the proof of Theorem 6.3 and prove a "relative" version of Conjecture 2.1 for pairs 
$\left(W, W_{L}\right)$, where now $W$ is any finite Coxeter group and $W_{L}$ is a parabolic subgroup of $W$, all of whose irreducible factors are of type $A$. If the conclusion of Theorem 7.1 were to hold for every parabolic subgroup $W$, not just those that are products of symmetric groups, then Conjecture 2.1 would hold for $W$. The statement of the conjecture, along with an expository review of the background material from the theories of Coxeter groups and hyperplane arrangements we use later in the paper, is given in $₫ 2$.

The subrepresentations of the right regular representation of $W$ that we consider arise from a decomposition of a subalgebra of the group algebra of $W$ known as "Solomon's descent algebra", into projective, indecomposable modules. Projective, indecomposable modules in an artinian $\mathbb{C}$-algebra are generated by idempotents. The idempotents in the descent algebra we use in this paper were discovered by Bergeron, Bergeron, Howlett, and Taylor [4]; we call them BBHT idempotents. In $\$ 3$ we study the relationships between BBHT idempotents for $W$ and BBHT idempotents for parabolic subgroups. We also compare the BBHT idempotents for $W$ with those of its irreducible factors when $W$ is reducible. In $\$ 4$ we show that the right ideals in the group algebra of $W$ generated by BBHT idempotents afford induced representations. It then follows that the BBHT idempotents give rise to a decomposition of the group algebra that is the direct analog of the decomposition of $H^{*}\left(M_{W}\right)$ given by Brieskorn's Lemma [11, Lemme 3].

The proof of Conjecture 2.1 for symmetric groups is given in $\$ 5$ and $\$ 6$. As a consequence, we obtain a decomposition of the group algebra $\mathbb{C} S_{n}$ as a direct sum of representations induced from one-dimensional representations of centralizers, one for each conjugacy class. Similar decompositions of the group algebra of the symmetric group have been proven independently by Bergeron, Bergeron, and Garsia [3], Hanlon [19], and more recently, Schocker [33], all using different methods.

For readers familiar with the literature on the free Lie algebra and its connections with the descent algebras and group algebras of symmetric groups, Theorems 5.1(a) and 6.3 (a) will seem familiar. Indeed, Theorem 5.1(a) was likely known in some form to Brandt [10, Wever [39, and Klyachko 22, and a proof of Theorem 6.3(a) can be extracted from results in [3, §4], 31, Theorem 8.24], and [15, §4]. In contrast with these references, where the methods are combinatorial and the emphasis is on the connections between the group algebra $\mathbb{C} S_{n}$ and the free Lie algebra on $n$ letters, our methods are mainly group- and representation-theoretic, using the theory of Coxeter groups in conjunction with special features of symmetric groups, and our focus is on the connections between the group algebra $\mathbb{C} S_{n}$ and the representations of $S_{n}$ on the cohomology spaces $H^{p}\left(M_{W}\right)$. Moreover, our line of reasoning, which is motivated by the arguments in Lehrer and Solomon [26], demonstrates a striking parallelism between the Orlik-Solomon algebras and group algebras of symmetric groups that to our knowledge has not been observed before. We hope that the approach taken in this paper will lead to a deeper understanding of the topological and geometric properties of general Coxeter arrangements. For example, as we show in $\$ 7$ the constructions in $\$ 5$ and $\$ 6$ have natural extensions when the focus is shifted from considering not just a single symmetric group to considering a product of symmetric groups embedded as a parabolic subgroup in a larger finite Coxeter group $W$, where the type of $W$ is arbitrary.

Bergeron and Bergeron conjecture in 2] that there might be a decomposition of the group algebra $\mathbb{C} W\left(B_{n}\right)$ analogous to the decomposition of $\mathbb{C} S_{n}$ studied by 
Bergeron-Bergeron-Garsia [3] and Hanlon [19]. In [5] Bergeron gives a decomposition of the group algebra of a hyperoctahedral group as a direct sum of representations induced from linear characters of subgroups. However, this decomposition is not the decomposition proposed in Conjecture 2.1] it is the analog for group algebras of hyperoctahedral groups of the decomposition of $H^{*}\left(M_{W}\right)$ found in [12].

\section{The Orlik-Solomon Algebra And Solomon's Descent Algebra}

In the rest of this paper we assume that $V$ is a finite-dimensional, complex vector space and $W \subseteq \mathrm{GL}(V)$ is a finite Coxeter group with Coxeter generating set $S$. Then each $s$ in $S$ acts on $V$ as a reflection with order two, and $W$ is generated by $S$ subject to the relations $(s t)^{m_{s, t}}=1$, where $m_{s, s}=1$ and $m_{s, t}=m_{t, s}>1$ for $s \neq t$ in $S$. Let $T$ denote the set of all reflections in $W$.

We assume also that a positive definite, Hermitian form $\langle\cdot, \cdot\rangle$ on $V$ is given and that $W$ is a subgroup of the unitary group of $V$ with respect to this form. It is known that $\operatorname{Fix}(W)^{\perp}$, the orthogonal complement of the space of fixed points of $W$ on $V$, has a basis $\Delta=\left\{\alpha_{s} \mid s \in S\right\}$ so that $\left\langle\alpha_{s}, \alpha_{t}\right\rangle=-\cos \left(\pi / m_{s, t}\right)$ for $s$ and $t$ in $S$. Then $s$ acts on $V$ as the reflection through the hyperplane orthogonal to $\alpha_{s}$, and $\Phi=\left\{w\left(\alpha_{s}\right) \mid w \in W, s \in S\right\}$ is a root system in $\operatorname{Fix}(W)^{\perp}$ with base $\Delta$.

2.1. Shapes and conjugacy classes. We begin by recalling a parameterization of the conjugacy classes in $W$ due to Geck and Pfeiffer (see [18, §3.2]) in a form compatible with the arrangement $(V, \mathcal{A})$ of $W$.

The lattice of $\mathcal{A}$, denoted by $L(\mathcal{A})$, is the set of subspaces of $V$ that arise as intersections of hyperplanes in $\mathcal{A}$ :

$$
L(\mathcal{A})=\left\{H_{t_{1}} \cap H_{t_{2}} \cap \cdots \cap H_{t_{p}} \mid t_{1}, t_{2}, \ldots, t_{p} \in T\right\} .
$$

For $X$ in $L(\mathcal{A})$ define

$$
W_{X}=\{w \in W \mid X \subseteq \operatorname{Fix}(w)\}
$$

to be the pointwise stabilizer of $X$ in $W$. It follows from Steinberg's Theorem 37 . that $W_{X}$ is generated by $\left\{t \in T \mid X \subseteq H_{t}\right\}$. It then follows that $X=\operatorname{Fix}\left(W_{X}\right)$, and so the assignment $X \mapsto W_{X}$ defines an injection from $L(\mathcal{A})$ to the set of subgroups of $W$. Notice that $W_{X}$ is again a Coxeter group.

The action of $W$ on $\mathcal{A}$ induces an action of $W$ on $L(\mathcal{A})$. Obviously, $w W_{X} w^{-1}=$ $W_{w(X)}$, and so for $X$ and $Y$ in $L(\mathcal{A})$ the subgroups $W_{X}$ and $W_{Y}$ are conjugate if and only if $X$ and $Y$ lie in the same $W$-orbit. Thus, the assignment $X \mapsto W_{X}$ induces a bijection between the set of orbits of $W$ on $L(\mathcal{A})$ and the set of conjugacy classes of subgroups $W_{X}$.

By a shape of $W$ we mean a $W$-orbit in $L(\mathcal{A})$. We denote the set of shapes of $W$ by $\Lambda$. For example, if $W$ is the symmetric group $S_{n}$, then $\Lambda$ is in bijection with the set of partitions of $n$ and with the set of Young diagrams with $n$ boxes. When $\lambda$ is a shape and $X$ is a subspace in $\lambda$, we say $\lambda$ is the shape of $W_{X}$.

It is shown in [30, §6.2] that the assignment $w \mapsto \operatorname{Fix}(w)$ defines a surjection from $W$ to $L(\mathcal{A})$. Composing with the map that sends an element $X$ in $L(\mathcal{A})$ to its $W$-orbit, we get a map

$$
\text { sh: } W \rightarrow \Lambda \text {. }
$$

We say $\operatorname{sh}(w)$ is the shape of $w$. Thus, $\operatorname{sh}(w)$ is the $W$-orbit of $\operatorname{Fix}(w)$ in $L(\mathcal{A})$. Clearly, sh is constant on conjugacy classes, and so we can define the shape of a conjugacy class to be the shape of any of its elements. 
An element $w$ in $W$, or its conjugacy class, is called cuspidal if $\operatorname{Fix}(w)=\operatorname{Fix}(W)$. For example, if $W$ is the symmetric group $S_{n}$, then the conjugacy class consisting of $n$-cycles is the only cuspidal class. In general, there is more than one cuspidal conjugacy class. Cuspidal elements and conjugacy classes are called elliptic by some authors.

Suppose that $\lambda$ is a shape, $X$ in $L(\mathcal{A})$ has shape $\lambda$, and $C$ is a conjugacy class in $W$ with shape $\lambda$. If $w$ is in $C$, then $\operatorname{Fix}(w)$ is in the $W$-orbit of $X$, and so $C \cap W_{X}$ is a non-empty union of cuspidal $W_{X}$-conjugacy classes. Geck and Pfeiffer [18, §3.2] have shown that in fact $C \cap W_{X}$ is a single $W_{X}$-conjugacy class. It follows that $C \mapsto C \cap W_{X}$ defines a bijection between the set of conjugacy classes in $W$ with shape $\lambda$ and the set of cuspidal conjugacy classes in $W_{X}$.

Fix a set $\left\{X_{\lambda} \mid \lambda \in \Lambda\right\}$ of $W$-orbit representatives in $L(\mathcal{A})$. Summarizing the preceding discussion we see that conjugacy classes in $W$ are parametrized by pairs $\left(\lambda, C_{\lambda}\right)$, where $\lambda$ is a shape and $C_{\lambda}$ is a cuspidal conjugacy class in $W_{X_{\lambda}}$.

2.2. The Orlik-Solomon algebra. Next, consider the cohomology ring $H^{*}\left(M_{W}\right)$. Arnold and Brieskorn (see [30, §1.1]) have computed $H^{*}\left(M_{W}\right)$. In the following we use the presentation of this algebra given by Orlik and Solomon [28].

Recall that the set $T$ of reflections in $W$ parametrizes the hyperplanes in $\mathcal{A}$. The Orlik-Solomon algebra of $W$ is the $\mathbb{C}$-algebra, $A=A(\mathcal{A})$, with generators $\left\{a_{t} \mid t \in T\right\}$ and relations

- $a_{t_{1}} a_{t_{2}}=-a_{t_{2}} a_{t_{1}}$ for $t_{1}$ and $t_{2}$ in $T$ and

- $\sum_{i=1}^{p}(-1)^{i} a_{t_{1}} \cdots \widehat{a_{t_{i}}} \cdots a_{t_{p}}=0$ whenever $\left\{H_{t_{1}}, \ldots, H_{t_{p}}\right\}$ is a linearly dependent subset of $\mathcal{A}$.

The algebra $A$ is a skew-commutative, graded, connected $\mathbb{C}$-algebra that is isomorphic as a graded algebra to $H^{*}\left(M_{W}\right)$. Let $A^{p}$ denote the degree $p$ subspace of $A$. Then

- $A^{0} \cong \mathbb{C}$

- for $1 \leq p \leq|S|$ the subspace $A^{p}$ is spanned by the set of all $a_{t_{1}} \cdots a_{t_{p}}$, where $\operatorname{codim} H_{t_{1}} \cap \cdots \cap H_{t_{p}}=p$, and

- $A^{p}=0$ for $p>|S|$.

See [30, §3.1] for details.

The action of $W$ on $\mathcal{A}$ extends to an action of $W$ on $A$ as algebra automorphisms. An element $w$ in $W$ acts on a generator $a_{t}$ of $A$ by $w a_{t}=a_{w t w^{-1}}$. With this $W$ action $A$ is isomorphic to $H^{*}\left(M_{W}\right)$ as graded $W$-algebras.

Orlik and Solomon 29] have shown that the normalizer of $W_{X}$ in $W$ is the setwise stabilizer of $X$ in $W$; that is,

$$
N_{W}\left(W_{X}\right)=\{w \in W \mid w(X)=X\} .
$$

For $X$ in $L(\mathcal{A})$, define $A_{X}$ to be the span of $\left\{a_{t_{1}} \cdots a_{t_{r}} \mid H_{t_{1}} \cap \cdots \cap H_{t_{r}}=X\right\}$. Proofs of the following statements may be found in [30, Corollary 3.27 and Theorem 6.27]:

- If $\operatorname{codim} X=p$, then $A_{X} \subseteq A^{p}$.

- There are vector space decompositions $A^{p} \cong \bigoplus_{\operatorname{codim} X=p} A_{X}$ and $A \cong$ $\bigoplus_{X \in L(\mathcal{A})} A_{X}$.

- For $w$ in $W, w A_{X}=A_{w(X)}$. Thus, $A_{X}$ is an $N_{W}\left(W_{X}\right)$-stable subspace of A. 
For a shape $\lambda$ in $\Lambda$, set $A_{\lambda}=\bigoplus_{X \in \lambda} A_{X}$. Suppose $X$ is a fixed subspace in $\lambda$ and that $\operatorname{codim} X=p$. Then $A_{\lambda}$ is a $W$-stable subspace of $A^{p}$ and we have isomorphisms of $\mathbb{C} W$-modules

$$
A_{\lambda} \cong \operatorname{Ind}_{N_{W}\left(W_{X}\right)}^{W}\left(A_{X}\right) \text { and } \quad A \cong \bigoplus_{\lambda \in \Lambda} A_{\lambda}
$$

(see [26]).

2.3. Solomon's descent algebra. In contrast with the Orlik-Solomon algebra $A$, which is defined for every complex reflection group, Solomon's descent algebra is defined using the Coxeter generating set $S$ of $W$ and so has no immediate analog for complex reflection groups that are not Coxeter groups.

Suppose that $I$ is a subset of $S$. Define

$$
X_{I}=\bigcap_{s \in I} H_{s} \quad \text { and } \quad W_{I}=\langle I\rangle .
$$

Then $X_{I}$ is in $L(\mathcal{A})$ and codim $X_{I}=|I|$. It follows from Steinberg's Theorem 37] that $W_{I}=W_{X_{I}}$. Recall that $\Delta=\left\{\alpha_{s} \mid s \in S\right\}$ is a base of $\Phi$. For $I \subseteq S$ define

$$
\Delta_{I}=\left\{\alpha_{s} \mid s \in I\right\} .
$$

Then $X_{I}$ is the orthogonal complement of the span of $\Delta_{I}$.

Orlik and Solomon (see [30, §6.2]) have shown that each orbit of $W$ on $L(\mathcal{A})$ contains a subspace $X_{I}$ for some subset $I$ of $S$. For subsets $I$ and $J$ of $S$ define $I \sim J$ if there is a $w$ in $W$ with $w\left(\Delta_{I}\right)=\Delta_{J}$. Then $\sim$ is an equivalence relation. It is well known that $W_{I}$ and $W_{J}$ are conjugate if and only if $I \sim J$ (see [35]). It follows that the assignment $I \mapsto X_{I}$ induces a bijection between the set of $\sim$ equivalence classes of subsets of $S$ and the set of shapes of $W$. We use this bijection to identify shapes of $W$ with subsets of the power set of $S$.

Next, let $\ell$ denote the length function of $W$ determined by the generating set $S$ and define

$$
W^{I}=\{w \in W \mid \ell(w s)>\ell(w) \forall s \in I\} .
$$

Then $W^{I}$ is a set of left coset representatives of $W_{I}$ in $W$. Also, define

$$
x_{I}=\sum_{w \in W^{I}} w
$$

in the group algebra $\mathbb{C} W$. Solomon 34 has shown that the span of $\left\{x_{I} \mid I \subseteq S\right\}$ is in fact a subalgebra of $\mathbb{C} W$. This subalgebra is denoted by $\Sigma(W)$ and is called the descent algebra of $W$. It is not hard to see that $\left\{x_{I} \mid I \subseteq S\right\}$ is linearly independent, and so $\operatorname{dim} \Sigma(W)=2^{|S|}$. Notice that $x_{S}=1$ is the identity in both $\mathbb{C} W$ and its subalgebra $\Sigma(W)$.

Bergeron, Bergeron, Howlett, and Taylor [4] have defined a basis $\left\{e_{I} \mid I \subseteq S\right\}$ of $\Sigma(W)$ that consists of quasi-idempotents and is compatible with the set of shapes of $W$. For $\lambda$ in $\Lambda$ define

$$
S_{\lambda}=\left\{I \subseteq S \mid X_{I} \in \lambda\right\} \quad \text { and } \quad e_{\lambda}=\sum_{I \in S_{\lambda}} e_{I}
$$


Then each $e_{\lambda}$ is idempotent and $\left\{e_{\lambda} \mid \lambda \in \Lambda\right\}$ is a complete set of primitive, orthogonal idempotents in $\Sigma(W)$. (See $\oint 3$ for more details.) In particular, $\sum_{\lambda \in \Lambda} e_{\lambda}=1$ in $\mathbb{C} W$.

Define $E_{\lambda}=e_{\lambda} \mathbb{C} W$. In $\$ 3$ we show that $e_{I} \mathbb{C} W_{I}$ affords an action of $N_{W}\left(W_{I}\right)$ and that if $I$ is in $S_{\lambda}$, then $E_{\lambda}$ is induced from $e_{I} \mathbb{C} W_{I}$. Thus, in analogy with the decomposition in $\$ 2.2$ of the Orlik-Solomon algebra $A$, we have isomorphisms of $\mathbb{C} W$-modules

$$
E_{\lambda} \cong \operatorname{Ind}_{N_{W}\left(W_{I}\right)}^{W}\left(e_{I} \mathbb{C} W_{I}\right) \quad \text { and } \quad \mathbb{C} W \cong \bigoplus_{\lambda \in \Lambda} E_{\lambda} .
$$

2.4. Centralizers and complements. The last ingredient we need in order to state the conjecture is a certain set of characters of centralizers of elements of $W$. These characters, together with the sign character, should quantify the difference between the representation of $W$ on $H^{p}\left(M_{W}\right)$ and a subrepresentation of the regular representation. They naturally arise in work of Howlett and Lehrer [21] and in recent results of Konvalinka, Pfeiffer, and Röver 23 that describe the structure of the centralizer of an element in $W$.

Suppose that $I$ is a subset of $S$ and $C$ is a conjugacy class in $W$ such that $C \cap W_{I}$ is a cuspidal conjugacy class in $W_{I}$. Howlett [20] has shown that $W_{I}$ has a complement, $N_{I}$, in $N_{W}\left(W_{I}\right)$. Moreover, it is shown in [23] that if $c$ is in $C \cap W_{I}$, then $Z_{W}(c) \subseteq N_{W}\left(W_{I}\right)$ and $Z_{W}(c) W_{I}=N_{W}\left(W_{I}\right)$. It follows that

(1) for any $X$ in $L(\mathcal{A}), W_{X}$ has a complement, say $N_{X}$, in $N_{W}\left(W_{X}\right)$ such that

$$
N_{W}\left(W_{X}\right) \cong W_{X} \rtimes N_{X}
$$

and

(2) for $c$ in $W_{X}$ cuspidal, $Z_{W}(c) \subseteq N_{W}\left(W_{X}\right)$ and $Z_{W}(c) / Z_{W_{X}}(c) \cong N_{X}$.

Recall $N_{W}\left(W_{X}\right)$ is the setwise stabilizer of $X$ in $W$. Define $\alpha_{X}: N_{W}\left(W_{X}\right) \rightarrow \mathbb{C}$ by $\alpha_{X}(n)=\left.\operatorname{det} n\right|_{X}$. For $c$ in $W$ with $X=\operatorname{Fix}(c)$, define $\alpha_{c}=\left.\alpha_{X}\right|_{Z_{W}(c)}$. Then

$$
\alpha_{c}(z)=\left.\operatorname{det} z\right|_{\operatorname{Fix}(c)}
$$

for $z$ in $Z_{W}(c)$.

2.5. Relating the Orlik-Solomon algebra and the descent algebra. We now have all the concepts we need in order to state the conjecture.

Let $\epsilon$ denote the sign character of $W$. For $c$ in $W$, define $X_{c}=\operatorname{Fix}(c), \operatorname{rk}(c)=$ $\operatorname{codim} X_{c}$, and $W_{c}=W_{X_{c}}$. Notice that $c$ is a cuspidal element in $W_{c}$.

Associated with each $\lambda$ in $\Lambda$ we have the $W$-stable subspace $A_{\lambda}$ of the OrlikSolomon algebra $A$, the right ideal $E_{\lambda}$ in $\mathbb{C} W$, and the set of conjugacy classes with shape $\lambda$. We conjecture that $A_{\lambda}$ and $E_{\lambda}$ are related to the set of conjugacy classes with shape $\lambda$ as follows.

Conjecture 2.1. Choose a set $\mathcal{C}$ of conjugacy class representatives in $W$. For $\lambda$ in $\Lambda$, set $\mathcal{C}_{\lambda}=\{c \in \mathcal{C} \mid \operatorname{sh}(c)=\lambda\}$. Then, for each conjugacy class representative $c$ in $\mathcal{C}$, there is a linear character $\varphi_{c}$ of $Z_{W}(c)$ such that for every $\lambda$ in $\Lambda$,

(a) the character of $E_{\lambda}$ is

$$
\sum_{c \in \mathcal{C}_{\lambda}} \operatorname{Ind}_{Z_{W}(c)}^{W}\left(\varphi_{c}\right)
$$

and 
(b) the character of $A_{\lambda}$ is

$$
\sum_{c \in \mathcal{C}_{\lambda}} \operatorname{Ind}_{Z_{W}(c)}^{W}\left(\epsilon_{c} \alpha_{c} \varphi_{c}\right)
$$

where $\epsilon_{c}$ denotes the restriction of $\epsilon$ to $Z_{W}(c)$.

In particular,

$$
H^{p}\left(M_{W}\right) \cong A^{p} \cong \bigoplus_{\operatorname{rk}(c)=p} \operatorname{Ind}_{Z_{W}(c)}^{W}\left(\epsilon_{c} \alpha_{c} \varphi_{c}\right)
$$

for $0 \leq p \leq|S|$,

$$
\mathbb{C} W \cong \bigoplus_{c \in \mathcal{C}} \operatorname{Ind}_{Z_{W}(c)}^{W}\left(\varphi_{c}\right), \quad \text { and } \quad H^{*}\left(M_{W}\right) \cong \bigoplus_{c \in \mathcal{C}} \operatorname{Ind}_{Z_{W}(c)}^{W}\left(\epsilon_{c} \alpha_{c} \varphi_{c}\right) .
$$

As stated in the introduction, we prove Conjecture 2.1 for symmetric groups in 95 and 96 . The conjecture is known to be true for all Coxeter groups with rank up to eight ([13, 6], 7], 8]).

We in fact prove more than is stated in the conjecture. First, we show that the character $\varphi_{c}$ of $Z_{W}(c)$ may be chosen to be a trivial extension of a character of $Z_{W_{c}}(c)$. Second, we construct explicit $\mathbb{C} W$-module isomorphisms

$$
E_{\lambda} \cong \operatorname{Ind}_{Z_{W}(c)}^{W}\left(\varphi_{c}\right) \text { and } A_{\lambda} \cong \operatorname{Ind}_{Z_{W}(c)}^{W}\left(\epsilon_{c} \alpha_{c} \varphi_{c}\right)
$$

In $\$ 7$ we extend the constructions in $\$ 5$ and $\sqrt[6]{6}$ and show that if $W$ is any finite Coxeter group, $\lambda$ is in $\Lambda, c$ is in $W$ with $\operatorname{sh}(c)=\lambda$, and the irreducible components of $W_{c}$ are all of type $A$, then the character $\varphi_{c}$ of $Z_{W_{c}}(c)$ constructed in $₫ 6$ extends to a character $\widetilde{\varphi}_{c}$ of $Z_{W}(c)$. Moreover, we construct explicit $\mathbb{C} W$-module isomorphisms

$$
E_{\lambda} \cong \operatorname{Ind}_{Z_{W}(c)}^{W}\left(\widetilde{\varphi}_{c}\right) \quad \text { and } \quad A_{\lambda} \cong \operatorname{Ind}_{Z_{W}(c)}^{W}\left(\epsilon_{c} \alpha_{c} \widetilde{\varphi}_{c}\right)
$$

Note that with the given assumptions we have $\left|\mathcal{C}_{\lambda}\right|=1$, and so the sums in Conjecture 2.1 (a) and (b) reduce to a single summand. Also, in contrast with the case when the ambient group $W$ is a symmetric group and $\widetilde{\varphi}_{c}$ is the trivial extension, in the general case $\widetilde{\varphi}_{c}$ may not be the trivial extension of $\varphi_{c}$.

A direct proof of the conjecture involves finding suitable linear characters $\varphi_{c}$ of $Z_{W}(c)$. The computations in [6] and [7], as well as calculations in type $B$, show that some natural guesses about the characters $\varphi_{c}$ are not true. For example, $Z_{W}(c)$ acts on the eigenspaces of $c$ in $V$, and so the powers of the determinant character of $Z_{W}(c)$ acting on an eigenspace of $c$ are linear characters of $Z_{W}(c)$. An example in [6. §4] shows that it is not always possible to choose $\varphi_{c}$ to be one of these characters for any eigenspace.

On the other hand, suppose that $c$ is an involution. Then $Z_{W}(c)=N_{W}\left(W_{X_{c}}\right) \cong$ $W_{X_{c}} \rtimes N_{X_{c}}$. Let $\epsilon_{X_{c}}$ be the sign character of $W_{X_{c}}$. Then in all the cases that have been computed so far, it turns out that $\varphi_{c}$ may be chosen to be the "trivial" extension of $\epsilon_{W_{c}}$ to $Z_{W}(c)$ in the sense that $\varphi_{c}(w n)=\epsilon_{X_{c}}(w)$ for $w$ in $W_{X_{c}}$ and $n$ in $N_{X_{c}}$. The representations $\operatorname{Ind}_{Z_{W}(c)}^{W} \varphi_{c}$ play a role in understanding the characters of finite reductive groups ([24], [17]), and the corresponding representations of the Iwahori-Hecke algebra of $W$ in [27] play a role in the representation theory of complex reductive groups. The fact that these representations seem to be closely related to the representation of $W$ on $H^{*}\left(M_{W}\right)$ is quite mysterious. 


\section{BBHT IDEMPOTENTS}

In this section we collect several preliminary results about the descent algebra of $W$ and the BBHT idempotents.

For subsets $I, J$, and $K$ of $S$ define

$$
W^{I J}=\left(W^{I}\right)^{-1} \cap W^{J} \quad \text { and } \quad W^{I J K}=\left\{w \in W^{I J} \mid w^{-1}\left(\Delta_{I}\right) \cap \Delta_{J}=\Delta_{K}\right\} .
$$

Then $W^{I J}$ is the set of minimal length $\left(W_{I}, W_{J}\right)$-double coset representatives in $W$. Solomon [34] has shown that $x_{I} x_{J}=\sum_{K} a_{I J K} x_{K}$, where $a_{I J K}=\left|W^{I J K}\right|$.

Let $2^{S}$ denote the power set of $S$ and fix a function $\sigma: 2^{S} \rightarrow \mathbb{R}_{>0}$. Notice that $W^{K}=\left\{w \in W \mid w\left(\Delta_{K}\right) \subseteq \Phi^{+}\right\}$, where $\Phi^{+}$is the positive system determined by $\Delta$. Thus, for $J \subseteq K$ and $w$ in $W^{K}$ we have $w\left(\Delta_{J}\right) \subseteq \Phi^{+}$. For subsets $J$ and $K$ of $S$ define

$$
m_{J K}^{\sigma}=\sum_{\substack{w \in W^{K} \\ w(\Delta J) \subseteq \Delta}} \sigma\left({ }^{w} J\right) \quad \text { if } \quad J \subseteq K, \quad \text { and } \quad m_{J K}^{\sigma}=0 \quad \text { if } \quad J \nsubseteq K .
$$

Because $\sigma(I)>0$ for all subsets $I$ of $S$, we have $m_{J J}^{\sigma} \neq 0$ for all $J$, and so the system of equations

$$
x_{K}=\sum_{J \subseteq S} m_{J K}^{\sigma} e_{J}^{\sigma}, \quad K \subseteq S
$$

can be solved uniquely for $\left\{e_{J}^{\sigma} \mid J \subseteq S\right\}$. Define $n_{J K}^{\sigma}$ and $e_{K}^{\sigma}$ by

$$
e_{K}^{\sigma}=\sum_{J \subseteq S} n_{J K}^{\sigma} x_{J}
$$

Then $e_{K}^{\sigma}$ is in $\Sigma(W), n_{K K}^{\sigma}=\left(m_{K K}^{\sigma}\right)^{-1}$ for all subsets $K$ of $S$, and $n_{J K}^{\sigma}=0$ when $J \nsubseteq K$.

Bergeron, Bergeron, Howlett, and Taylor [4, §7] have shown that $e_{I}^{\sigma}$ is a quasiidempotent in $\Sigma(W)$. Precisely, for $\lambda$ in $\Lambda$ define $\sigma(\lambda)=\sum_{I \in S_{\lambda}} \sigma(I)$. Then

$$
e_{I}^{\sigma} e_{J}^{\sigma}=\sigma(\lambda)^{-1} e_{J}^{\sigma}
$$

when $I$ and $J$ are in $S_{\lambda}$. Thus, if we set

$$
e_{\lambda}^{\sigma}=\sum_{I \in S_{\lambda}} \sigma(I) e_{I}^{\sigma}
$$

it follows from (3.2) that $e_{\lambda}^{\sigma}$ is an idempotent in $\Sigma(W)$ and hence an idempotent in $\mathbb{C} W$. We call the quasi-idempotents $e_{I}^{\sigma}$ BBHT quasi-idempotents and the idempotents $e_{\lambda}^{\sigma}$ BBHT idempotents.

By definition we have $1=x_{S}=\sum_{J \subseteq S} n_{J S}^{\sigma} e_{J}^{\sigma}$, and so $1=\sum_{\lambda \in \Lambda} e_{\lambda}^{\sigma}$ in $\Sigma(W)$ and $\mathbb{C} W$. It follows that $\left\{e_{\lambda}^{\sigma} \mid \lambda \in \Lambda\right\}$ is a set of pairwise orthogonal idempotents in $\mathbb{C} W$ and that

$$
e_{\lambda}^{\sigma} e_{I}^{\sigma}=e_{I}^{\sigma} \quad \text { and } \quad e_{I}^{\sigma} e_{\lambda}^{\sigma}=\sigma(\lambda)^{-1} e_{\lambda}^{\sigma}
$$

for $I \in S_{\lambda}$.

In the special case when the function $\sigma(I)=1$ for all subsets $I$ of $S$ we do not include $\sigma$ in the notation. Thus,

$$
\begin{gathered}
m_{J K}=\left|\left\{w \in W^{K} \mid w\left(\Delta_{J}\right) \subseteq \Delta\right\}\right| \text { for } J \subseteq K, \\
e_{K}=\sum_{J \subseteq S} n_{J K} x_{J}, \quad \text { and } \quad e_{\lambda}=\sum_{I \in S_{\lambda}} e_{I} .
\end{gathered}
$$


Notice that the quantities $m_{J K}^{\sigma}, e_{J}^{\sigma}, m_{J K}, e_{J}, \ldots$ are defined relative to an ambient Coxeter system $(W, S)$. Below we also consider the analogous quantities defined relative to a parabolic subsystem $\left(W_{L}, L\right)$. To help keep things straight, in this section and the next we use the following conventions:

- $\sigma$ always denotes a function from $2^{S}$ to $\mathbb{R}_{>0}$.

- When $\sigma(I)=1$ for all $I \subseteq S$, the BBHT quasi-idempotents in $\mathbb{C} W$ defined with respect to $\sigma$ are denoted by $e_{J}$.

- $\tau$ always denotes a function from $2^{L}$ to $\mathbb{R}_{>0}$, where $L$ is a subset of $S$.

- When $\tau(I)=1$ for all $I \subseteq L$, the BBHT quasi-idempotents in $\mathbb{C} W_{L}$ defined with respect to $\tau$ are denoted by $e_{J}^{L}$. Thus, $e_{J}^{S}=e_{J}$.

For example, $\{S\}$ is a shape of $W$ and $\{L\}$ is a shape of $W_{L}$. Then $e_{\{S\}}^{\sigma}=\sigma(S) e_{S}^{\sigma}$, $e_{\{S\}}=e_{S}$, and $e_{L}^{L}=e_{L}^{\tau}=e_{\{L\}}^{\tau}$ when $\tau(I)=1$ for all subsets $I$ of $L$.

The following lemmas give some translation properties for the quantities defined above.

Lemma 3.1. Suppose that $K \subseteq S$ and $d$ is in $W$ with $d^{-1}\left(\Delta_{K}\right) \subseteq \Delta$. Then

(a) $x_{K^{d}}=x_{K} d$;

(b) $m_{I^{d} J^{d}}^{\sigma}=m_{I J}^{\sigma}$ for $I \subseteq J \subseteq K$; and

(c) $e_{L^{d}}^{\sigma}=e_{L}^{\sigma} d$ for $L \subseteq K$.

Proof. It is shown in [4, Lemma 2.4] that $W^{K^{d}}=W^{K} d$. Statement (a) follows immediately.

Suppose that $I \subseteq J \subseteq K$. Clearly, $\Delta_{L^{d}}=d^{-1}\left(\Delta_{L}\right)$ for all $L \subseteq K$, and so

$$
m_{I^{d} J^{d}}^{\sigma}=\sum_{\substack{w \in W^{J^{d}} \\ w\left(\Delta_{I^{d}}\right) \subseteq \Delta}} \sigma\left({ }^{w}\left(I^{d}\right)\right)=\sum_{\substack{w d^{-1} \in W^{J} \\ w d^{-1}\left(\Delta_{I}\right) \subseteq \Delta}} \sigma\left({ }^{-1} I\right)=\sum_{\substack{y \in W^{J} \\ y\left(\Delta_{I}\right) \subseteq \Delta}} \sigma\left({ }^{y} I\right)=m_{I J}^{\sigma} .
$$

This proves (b).

Using (a) and (b) we see that for $J \subseteq K$,

$$
\sum_{I} m_{I J}^{\sigma}\left(e_{I}^{\sigma} d\right)=x_{J} d=x_{J^{d}}=\sum_{I} m_{I J^{d}}^{\sigma} e_{I}^{\sigma}=\sum_{I} m_{d J}^{\sigma} e_{I}^{\sigma}=\sum_{I} m_{I J}^{\sigma} e_{I^{d}}^{\sigma} .
$$

Thus, $\sum_{I} m_{I J}^{\sigma}\left(e_{I}^{\sigma} d\right)=\sum_{I} m_{I J}^{\sigma} e_{I^{d}}^{\sigma}$. Now fix a subset $L$ of $K$, multiply both sides by $n_{J L}^{\sigma}$, and sum over $J$, to get $e_{L}^{\sigma} d=e_{L^{d}}^{\sigma}$. (Note that $n_{J L}^{\sigma}=0$ unless $J \subseteq L$.) This proves (c).

Recall that we have fixed a positive, definite, Hermitian form on $V$ such that $W$ is a subgroup of $\mathrm{U}(V)$, the unitary group of $V$. Define

$$
N(W)=\{n \in \mathrm{U}(V) \mid n(\Delta)=\Delta\} .
$$

Notice that if $n$ is in $N(W)$, then $n S n^{-1}=S$. Thus, $N(W)$ acts on $S$ and on $2^{S}$, and $W N(W) \cong W \rtimes N(W)$.

Lemma 3.2. Suppose that $n$ is in $N(W)$ and that $\sigma\left(I^{n}\right)=\sigma(I)$ for all $I \subseteq S$. Then $e_{I^{n}}^{\sigma}=n^{-1} e_{I}^{\sigma} n$ for $I \subseteq S$. In particular, $n$ centralizes $e_{S}^{\sigma}$ in $\mathbb{C} W$.

Proof. Because $n(\Delta)=\Delta$, it follows that $\ell\left(n w n^{-1}\right)=\ell(w)$ for all $w$ in $W$. Therefore, $n^{-1} W^{I} n=W^{I^{n}}$, and hence $n^{-1} x_{I} n=x_{I^{n}}$ for all $I \subseteq S$. 
Suppose $I \subseteq J \subseteq S$. Then

$$
\sum_{\substack{w \in W^{J^{n}} \\ w\left(\Delta_{I^{n}}\right) \subseteq \Delta}} \sigma\left({ }^{w}\left(I^{n}\right)\right)=\sum_{\substack{n w n^{-1} \in W^{J} \\ n w n^{-1}\left(\Delta_{I}\right) \subseteq \Delta}} \sigma\left(I^{n w^{-1}}\right)=\sum_{\substack{y \in W^{J} \\ y\left(\Delta_{I}\right) \subseteq \Delta}} \sigma\left(\left({ }^{y} I\right)^{n}\right)=\sum_{\substack{y \in W^{J} \\ y\left(\Delta_{I}\right) \subseteq \Delta}} \sigma\left({ }^{y} I\right),
$$

and so $m_{I^{n} J^{n}}^{\sigma}=m_{I J}^{\sigma}$. Thus, $n_{I^{n} J^{n}}^{\sigma}=n_{I J}^{\sigma}$ and it follows from (3.1) that $n^{-1} e_{I}^{\sigma} n=$ $e_{I^{n}}^{\sigma}$.

Lemma 3.3. Let $w_{0}$ denote the longest element in $W$.

(a) The element $w_{0}$ is in $\Sigma(W)$ and

$$
w_{0}=\sum_{L \subseteq S}(-1)^{|L|} e_{L}=\sum_{\lambda \in \Lambda}(-1)^{d_{\lambda}} e_{\lambda}
$$

where $d_{\lambda}=|L|$ for $L$ in $S_{\lambda}$.

(b) For $J \subseteq S, w_{0} e_{J}=(-1)^{|J|} e_{J}, w_{0} e_{J} w_{0}=e_{J} w_{0}$, and $e_{J} w_{0}=(-1)^{|J|} e_{J^{w_{0}}}$.

Proof. For $J \subseteq S$ define $Y^{J}=\{w \in W \mid\{s \in S \mid w s>w\}=J\}$. Then $W^{J}=\amalg_{J \subseteq K} Y^{K}$, and so $W^{J} \cap P=\amalg_{J \subseteq K}\left(Y^{K} \cap P\right)$ for every subset $P$ of $W$. For $P \subseteq W$ define

$$
p_{J}^{P}=\sum_{w \in W^{J} \cap P} w, \quad q_{K}^{P}=\sum_{w \in Y^{K} \cap P} w, \quad f_{J}^{P}=\left|W^{J} \cap P\right|, \quad \text { and } \quad g_{K}^{P}=\left|Y^{K} \cap P\right| .
$$

Then $p_{J}^{P}=\sum_{J \subseteq K} q_{K}^{P}$ and $f_{J}^{P}=\sum_{J \subseteq K} g_{K}^{P}$, and so by Möbius inversion,

$$
q_{J}^{P}=\sum_{J \subseteq K}(-1)^{|K|-|J|} p_{K}^{P} \quad \text { and } \quad g_{J}^{P}=\sum_{J \subseteq K}(-1)^{|K|-|J|} f_{K}^{P} .
$$

Taking $J=\emptyset$ and $P=W$ in (3.5), we have $Y^{\emptyset}=\left\{w_{0}\right\}$, and so

$$
w_{0}=q_{\emptyset}^{W}=\sum_{K}(-1)^{|K|} p_{K}^{W}=\sum_{K}(-1)^{|K|} x_{K} .
$$

For $J \subseteq S$, set $W_{+}^{J}=\left\{w \in W \mid w\left(\Delta_{J}\right) \subseteq \Delta\right\}$. Then for $J \subseteq K, m_{J K}=$ $\left|W^{K} \cap W_{+}^{J}\right|$. Taking $P=W_{+}^{J}$ in (3.5), we have $Y^{J} \cap W_{+}^{J}=\left\{w_{0} w_{J}\right\}$, where $w_{J}$ is the longest element in $W_{J}$, and so $g_{J}^{P}=1$ and $f_{K}^{P}=m_{J K}$. Therefore,

$$
(-1)^{|J|}=\sum_{J \subseteq K}(-1)^{|K|} m_{J K}
$$

Using (3.6) and (3.7), we have

$$
\begin{aligned}
w_{0}=\sum_{K}(-1)^{|K|} x_{K}=\sum_{K}(-1)^{|K|} \sum_{J} m_{J K} e_{J} & =\sum_{J}\left(\sum_{J \subseteq K}(-1)^{|K|} m_{J K}\right) e_{J} \\
& =\sum_{J}(-1)^{|J|} e_{J}=\sum_{\lambda \in \Lambda}(-1)^{d_{\lambda}} e_{\lambda} .
\end{aligned}
$$


Now suppose that $J \subseteq S$ and $\mu \in \Lambda$ are such that $J \in S_{\mu}$. By (3.3), we have $e_{\mu} e_{J}=e_{J}$, and so

$$
w_{0} e_{J}=\sum_{\lambda \in \Lambda}(-1)^{d_{\lambda}} e_{\lambda} e_{\mu} e_{J}=(-1)^{d_{\mu}} e_{\mu} e_{J}=(-1)^{|J|} e_{J} .
$$

For subsets $J$ and $K$ of $S$ we have $m_{J K}=m_{J^{w_{0}} K^{w_{0}}}$. Thus, $n_{J K}=n_{J^{w_{0}} K^{w_{0}}}$ and it follows from (3.1) that $w_{0} e_{J} w_{0}=e_{J} w_{0}$. Finally,

$$
e_{J} w_{0}=w_{0}\left(w_{0} e_{J} w_{0}\right)=(-1)^{\left|J^{w_{0}}\right|} e_{J^{w_{0}}}=(-1)^{|J|} e_{J} w_{0} .
$$

This completes the proof of the lemma.

For a subset $L$ of $S$, the pair $\left(W_{L}, L\right)$ is a Coxeter system. Because $W^{L}$ is a complete set of left coset representatives of $W_{L}$ in $W$, left multiplication by $x_{L}$ defines an embedding of $\mathbb{C} W_{L}$ into $\mathbb{C} W$. For $I \subseteq L$ define $W_{L}^{I}=W_{L} \cap W^{I}$ and $x_{I}^{L}=\sum_{w \in W_{L}^{I}} w$. Then $\left\{x_{I}^{L} \mid I \subseteq L\right\}$ is a basis of $\Sigma\left(W_{L}\right)$. It is well known and easy to prove that $W^{L} W_{L}^{I}=W^{I}$, and so $x_{L} x_{I}^{L}=x_{I}$. If $n$ is in $N_{L}$, then $n\left(\Delta_{L}\right)=\Delta_{L}$, and so by Lemma 3.1 (a) we have $x_{L} n=x_{L}$. It follows that $x_{L} \mathbb{C} W_{L}$ is stable under right multiplication by elements of $N_{W}\left(W_{L}\right)$.

It is straightforward to check that for $a$ in $\mathbb{C} W_{L}$ and $w n$ in $W_{L} N_{L}$, the assignment $(a, w n) \mapsto a \cdot w n=n^{-1} a w n$ defines a right action of the group $N_{W}\left(W_{L}\right)=W_{L} N_{L}$ on $\mathbb{C} W_{L}$. Then

$$
x_{L}(a \cdot w n)=x_{L} n^{-1} a w n=x_{L} a w n,
$$

and so left multiplication by $x_{L}$ defines an $N_{W}\left(W_{L}\right)$-equivariant embedding of $\mathbb{C} W_{L}$ into $\mathbb{C W}$. For later reference we record this fact in the following lemma.

Lemma 3.4. Suppose that $L$ is a subset of $S$. Then $N_{W}\left(W_{L}\right)$ acts on $\mathbb{C} W_{L}$ by $a \cdot w n=n^{-1}$ awn, for $a \in \mathbb{C} W_{L}$, w in $W_{L}$, and $n \in N_{L}$. Moreover, left multiplication by $x_{L}$ defines an $N_{W}\left(W_{L}\right)$-equivariant embedding of $\mathbb{C} W_{L}$ into $\mathbb{C} W$.

Recall that the quasi-idempotents $e_{I}^{\sigma}$ are defined relative to the ambient set $S$ and the function $\sigma$. Define

$$
\sigma_{L}: 2^{L} \rightarrow \mathbb{R}_{>0} \quad \text { by } \quad \sigma_{L}(I)=m_{I L}^{\sigma} .
$$

Then for $J \subseteq L$ we have the quasi-idempotent $e_{J}^{\sigma_{L}}=\sum_{I \subseteq L} n_{I J}^{\sigma_{L}} x_{I}^{L}$ in $\mathbb{C} W_{L}$ defined relative to the set $L$ and the function $\sigma_{L}$.

Lemma 3.5. Suppose that $I, J$, and $L$ are subsets of $S$ with $I, J \subseteq L$. Then

(a) $m_{I J}^{\sigma_{L}}=m_{I J}^{\sigma}$ and $n_{I J}^{\sigma_{L}}=n_{I J}^{\sigma}$;

(b) $x_{L} e_{J}^{\sigma_{L}}=e_{J}^{\sigma}$; and

(c) $n^{-1} e_{J}^{\sigma_{L}} n=e_{J^{n}}^{\sigma_{L}}$ for $n$ in $N_{L}$.

Proof. It is shown in [4, Theorem 7.5] that $m_{I J}^{\sigma_{L}}=m_{I J}^{\sigma}$. It then follows from the definitions that $n_{I J}^{\sigma_{L}}=n_{I J}^{\sigma}$. This proves (a). Now

$$
x_{L} e_{J}^{\sigma_{L}}=x_{L} \sum_{\substack{I \\ I \subseteq J}} n_{I J}^{\sigma_{L}} x_{I}^{L}=\sum_{\substack{I \subseteq J \\ I \subseteq J}} n_{I J}^{\sigma_{L}} x_{L} x_{I}^{L}=\sum_{\substack{I \\ I \subseteq J}} n_{I J}^{\sigma} x_{I}=e_{J}^{\sigma},
$$

and so (b) holds.

Suppose $n$ is in $N_{L}$. Then using (b) and Lemma 3.1 we have

$$
e_{J}^{\sigma} n=x_{L} e_{J}^{\sigma_{L}} n=\left(x_{L} n\right)\left(n^{-1} e_{J}^{\sigma_{L}} n\right)=x_{L}\left(n^{-1} e_{J}^{\sigma_{L}} n\right)
$$


and

$$
e_{J}^{\sigma} n=e_{J^{n}}^{\sigma}=x_{L^{n}} e_{J^{n}}^{\sigma_{L}}=x_{L} e_{J^{n}}^{\sigma_{L}}
$$

Now $n^{-1} e_{J}^{\sigma_{L}} n$ and $e_{J^{n}}^{\sigma_{L}}$ are both in $\mathbb{C} W_{L}$ and $x_{L}\left(n^{-1} e_{J}^{\sigma_{L}} n\right)=x_{L} e_{J^{n}}^{\sigma_{L}}$, so we conclude from Lemma 3.4 that $n^{-1} e_{J}^{\sigma_{L}} n=e_{J^{n}}^{\sigma_{L}}$. This proves (c).

We conclude this section with a description of the quasi-idempotents $e_{K}^{\sigma}$ in the case when $W$ is reducible.

Suppose that $W$ is reducible, say $W \cong W_{1} \times W_{2}$. Then $S=S_{1} \amalg S_{2}$ is the disjoint union of $S_{1}$ and $S_{2}$, where $W_{1}=\left\langle S_{1}\right\rangle$ and $W_{2}=\left\langle S_{2}\right\rangle$, and $\Delta=\Delta_{1} \amalg \Delta_{2}$, where $\Delta_{i}=\left\{\alpha \mid s_{\alpha} \in S_{i}\right\}$ for $i=1,2$.

Suppose $K$ is a subset of $S$. Then $K=K_{1} \amalg K_{2}$, where $K_{i}=K \cap S_{i}$ for $i=1,2$. Every element in $W$ has a unique expression as a product $w_{1} w_{2}$ with $w_{1}$ in $W_{1}$ and $w_{2}$ in $W_{2}$. Then $w_{1} w_{2}$ is in $W_{K}$ if and only if $w_{1}$ is in $W_{K_{1}}$ and $w_{2}$ is in $W_{K_{2}}$. Moreover, $w_{1} w_{2}$ is in $W^{K}$ if and only if $w_{1}$ is in $W^{K_{1}}$ and $w_{2}$ is in $W^{K_{2}}$. It follows that $x_{K}=x_{K_{1}} x_{K_{2}}$. If $J$ is a subset of $K$, then $\Delta_{J}=\Delta_{J_{1}} \amalg \Delta_{J_{2}}$ and $w_{1} w_{2}\left(\Delta_{J}\right) \subseteq \Delta$ if and only if $w_{1}\left(\Delta_{J_{1}}\right) \subseteq \Delta_{1}$ and $w_{2}\left(\Delta_{J_{2}}\right) \subseteq \Delta_{2}$. Similarly, $w_{1} w_{2}\left(J_{1} \amalg J_{2}\right)={ }^{w_{1}} J_{1} \amalg{ }^{w_{2}} J_{2}$.

Now suppose that $\sigma: 2^{S} \rightarrow \mathbb{R}_{>0}$ has the property that $\sigma\left(J_{1} \amalg J_{2}\right)=\sigma\left(J_{1}\right) \sigma\left(J_{2}\right)$ for $J_{1} \subseteq S_{1}$ and $J_{2} \subseteq S_{2}$. Then for $J \subseteq K \subseteq S$ we have

$$
\begin{aligned}
m_{J K}^{\sigma}= & \sum_{\substack{w \in W^{K} \\
w\left(\Delta_{J}\right) \subseteq \Delta}} \sigma\left({ }^{w} J\right) \\
= & \sum_{\substack{w_{1} \in W^{K_{1}}, w_{2} \in W^{K_{2}} \\
w_{1}\left(\Delta_{J_{1}}\right) \subseteq \Delta_{1}, w_{2}\left(\Delta_{J_{2}}\right) \subseteq \Delta_{2}}} \sigma\left({ }^{w_{1}} J_{1} \amalg^{w_{2}} J_{2}\right) \\
= & \sum_{\substack{w_{1} \in W^{K_{1}} \\
w_{1}\left(\Delta_{J_{1}}\right) \subseteq \Delta_{1}}} \sum_{w_{2}\left(\Delta_{J_{2}} \in W^{K_{2}} \subseteq \Delta_{2}\right.} \sigma\left({ }^{w_{1}} J_{1}\right) \sigma\left({ }^{w_{2}} J_{2}\right) \\
= & m_{J_{1} K_{1}}^{\sigma} m_{J_{2} K_{2}}^{\sigma} \\
= & m_{J_{1} K_{1}}^{\sigma_{1}} m_{J_{2} K_{2}}^{\sigma_{2}},
\end{aligned}
$$

where $\sigma_{i}$ is the restriction of $\sigma$ to $2^{S_{i}}$ for $i=1,2$. Conversely, if we are given functions $\sigma_{i}: 2^{S_{i}} \rightarrow \mathbb{R}_{>0}$ for $i=1,2$ and define $\sigma: 2^{S} \rightarrow \mathbb{R}_{>0}$ by $\sigma(K)=\sigma_{1}\left(K_{1}\right) \sigma_{2}\left(K_{2}\right)$, then $m_{J_{1} K_{1}}^{\sigma_{1}} m_{J_{2} K_{2}}^{\sigma_{2}}=m_{J_{1} K_{1}}^{\sigma} m_{J_{2} K_{2}}^{\sigma}=m_{J K}^{\sigma}$.

With $\sigma$ as above, set $f_{J}^{\sigma}=e_{J_{1}}^{\sigma} e_{J_{2}}^{\sigma}$ for $J=J_{1} \amalg J_{2} \subseteq S$. Then

$$
\sum_{J \subseteq K} m_{J K}^{\sigma} f_{J}^{\sigma}=\sum_{\substack{J_{1} \subseteq K_{1} \\ J_{2} \subseteq K_{2}}} m_{J_{1} K_{1}}^{\sigma} m_{J_{2} K_{2}}^{\sigma} e_{K_{1}}^{\sigma} e_{K_{2}}^{\sigma}=x_{K_{1}} x_{K_{2}}=x_{K}
$$

and so $f_{J}^{\sigma}=e_{J}^{\sigma}$. This proves the following proposition.

Proposition 3.6. Suppose $W \cong W_{1} \times W_{2}$ is reducible and $S=S_{1} \amalg S_{2}$, where $W_{1}=\left\langle S_{1}\right\rangle$ and $W_{2}=\left\langle S_{2}\right\rangle$. Suppose in addition that $\sigma: 2^{S} \rightarrow \mathbb{R}_{>0}$ has the property that for $J \subseteq S, \sigma(J)=\sigma\left(J \cap S_{1}\right) \sigma\left(J \cap S_{2}\right)$. Then

$$
e_{J}^{\sigma}=e_{J \cap S_{1}}^{\sigma} e_{J \cap S_{2}}^{\sigma} \quad \text { and } \quad e_{J}=e_{J \cap S_{1}} e_{J \cap S_{2}} .
$$




\section{4. $E_{\lambda}$ IS AN INDUCED REPRESENTATION}

Suppose $\lambda$ is in $\Lambda$ and $\sigma: 2^{S} \rightarrow \mathbb{R}_{>0}$. Define

$$
E_{\lambda}^{\sigma}=e_{\lambda}^{\sigma} \mathbb{C} W
$$

to be the right ideal in $\mathbb{C} W$ generated by $e_{\lambda}^{\sigma}$. Similarly, using the notation in (3.4), define

$$
E_{\lambda}=e_{\lambda} \mathbb{C} W .
$$

We have seen in $₫ 2.2$ that $A_{\lambda} \cong \operatorname{Ind}_{N_{W}\left(W_{X}\right)}^{W}\left(A_{X}\right)$ for $X$ in $\lambda$. In this section we show that $E_{\lambda}^{\sigma}$ has a similar description as an induced representation, and we analyze how $E_{\lambda}^{\sigma}$ depends on the choice of $\sigma$. In particular, it is shown in Corollary 4.8 that for $L$ in $S_{\lambda}$,

$$
E_{\lambda} \cong \operatorname{Ind}_{N_{W}\left(W_{L}\right)}^{W}\left(e_{L} \mathbb{C} W_{L}\right) \cong \operatorname{Ind}_{N_{W}\left(W_{L}\right)}^{W}\left(e_{L}^{L} \mathbb{C} W_{L}\right)
$$

We begin with a lemma which follows immediately from (3.3).

Lemma 4.1. Suppose that $\lambda$ is in $\Lambda$ and $I$ is in $S_{\lambda}$. Then $E_{\lambda}^{\sigma}=e_{I}^{\sigma} \mathbb{C} W$.

The next proposition shows that up to isomorphism, $E_{\lambda}^{\sigma}$ does not depend on $\sigma$.

Proposition 4.2. Suppose that $\lambda$ is in $\Lambda$ and that $\sigma$ and $\sigma_{1}$ are functions from $2^{S}$ to $\mathbb{R}_{>0}$. Then there is a unit $u$ in $\Sigma(W)$ such that left multiplication by $u$ defines an isomorphism of right $\mathbb{C} W$-modules $E_{\lambda}^{\sigma} \cong E_{\lambda}^{\sigma_{1}}$.

Proof. Let $\operatorname{rad}(\Sigma(W))$ denote the Jacobson radical of $\Sigma(W)$ and let $\theta$ denote the natural projection from $\Sigma(W)$ to $\Sigma(W) / \operatorname{rad}(\Sigma(W))$. Bergeron, Bergeron, Howlett, and Taylor [4, §7] have shown that $\theta\left(e_{\lambda}^{\sigma}\right)=\theta\left(e_{\lambda}^{\sigma_{1}}\right)$ is a primitive idempotent in $\Sigma(W) / \operatorname{rad}(\Sigma(W))$. Thus, it follows from [38, Theorem 3.1] that there is a unit $u$ in $1+\operatorname{rad}(\Sigma(W))$ such that $u e_{\lambda}^{\sigma}=e_{\lambda}^{\sigma_{1}} u$. Then left multiplication by $u$ defines an isomorphism of right $\mathbb{C} W$-modules $e_{\lambda}^{\sigma} \mathbb{C} W \cong e_{\lambda}^{\sigma_{1}} \mathbb{C} W$.

Suppose that $N$ is a subgroup of $N(W)$, so $W N$ is a subgroup of $N_{\mathrm{U}(V)}(W)$. Then as in Lemma 3.4. $W N$ acts on $\mathbb{C} W$ on the right by $a \cdot w n=n^{-1}$ awn for $a$ in $\mathbb{C} W, w$ in $W$, and $n$ in $N$. If $N$ centralizes $e_{\lambda}^{\sigma}$, then clearly $E_{\lambda}^{\sigma}$ is a $W N$-submodule of $\mathbb{C} W$. It follows from Lemma 3.2 that if $\sigma$ is constant on $N$-orbits in $2^{S}$, then $N$ centralizes $e_{\{S\}}^{\sigma}=e_{S}^{\sigma}$. More generally, if $\sigma$ is constant on $N$-orbits in $2^{S}$ and $S_{\lambda}$ is $N$-stable, then $N$ centralizes $e_{\lambda}^{\sigma}$.

Let $\Sigma(W)^{N}$ denote the algebra of $N$-invariants in $\Sigma(W)$.

Proposition 4.3. Suppose that $\lambda$ is in $\Lambda$ and that $\sigma$ and $\sigma_{1}$ are functions from $2^{S}$ to $\mathbb{R}_{>0}$ such that $S_{\lambda}$ is $N$-stable, $N$ centralizes $e_{\lambda}^{\sigma}$, and $\sigma_{1}$ is constant on $N$-orbits in $2^{S}$. Then there is a unit $v$ in $\Sigma(W)^{N}$ such that left multiplication by $v$ defines an isomorphism of right $\mathbb{C} W N$-modules $E_{\lambda}^{\sigma}$ and $E_{\lambda}^{\sigma_{1}}$.

Proof. We saw in the proof of Proposition 4.2 that there is a unit $u$ in $1+\operatorname{rad}(\Sigma(W))$ such that $u e_{\lambda}^{\sigma}=e_{\lambda}^{\sigma_{1}} u$, and we observed in the proof of Lemma 3.2 that $n^{-1} x_{I} n=$ $x_{I^{n}}$ for $I \subseteq S$ and $n$ in $N$. It follows that $\Sigma(W)$ and $\operatorname{rad}(\Sigma(W))$ are stable under conjugation by $N$, and so $v=\frac{1}{|N|} \sum_{n \in N} n^{-1}$ un is in $1+\operatorname{rad}(\Sigma(W))$ and hence is a unit. By assumption, $N$ centralizes $e_{\lambda}^{\sigma}$ and $e_{\lambda}^{\sigma_{1}}$ and it follows that $v e_{\lambda}^{\sigma}=$ $e_{\lambda}^{\sigma_{1}} v$. Therefore, left multiplication by $v$ defines an $N_{\mathrm{U}(V)}(W)$-module isomorphism $e_{\lambda}^{\sigma} \mathbb{C} W \cong e_{\lambda}^{\sigma_{1}} \mathbb{C} W$. 
In the next proposition, $\ell_{x}$ denotes left multiplication by $x$.

Proposition 4.4. Suppose $L$ is a subset of $S, \sigma: 2^{S} \rightarrow \mathbb{R}_{>0}, \tau: 2^{L} \rightarrow \mathbb{R}_{>0}$, and $\tau$ is constant on $N_{L}$-orbits. Then there is a unit $v$ in $\Sigma\left(W_{L}\right)^{N_{L}}$ such that if $\mu_{v}=\ell_{x_{L}} \ell_{v} \ell_{x_{L}}^{-1}$, then the diagram

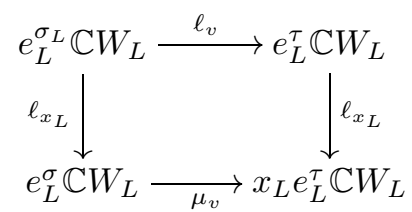

is a commutative diagram of right $N_{W}\left(W_{L}\right)$-modules and $N_{W}\left(W_{L}\right)$-isomorphisms.

Proof. It follows from Lemma 3.5 (c) and Lemma 3.2 that $e_{L}^{\sigma_{L}} \mathbb{C} W_{L}$ and $e_{L}^{\tau} \mathbb{C} W_{L}$ are $N_{W}\left(W_{L}\right)$-stable right ideals of $\mathbb{C} W_{L}$, where $N_{W}\left(W_{L}\right)$ acts on $\mathbb{C} W_{L}$ as in Lemma 3.4. By Lemma 3.5(b) we have $e_{L}^{\sigma}=x_{L} e_{L}^{\sigma_{L}}$, and so it follows from Lemma 3.1(a) that $e_{L}^{\sigma} \mathbb{C} W_{L}$ and $x_{L} e_{L}^{\tau} \mathbb{C} W_{L}$ are stable under right multiplication by $N_{W}\left(W_{L}\right)$. It now follows from Lemma 3.4 that the vertical maps are isomorphisms of $N_{W}\left(W_{L}\right)$ modules.

The hypotheses of Proposition 4.3 are satisfied with $L,\{L\}$, and $\sigma_{L}$ in place of $S, S_{\lambda}$, and $\sigma$. Thus, there is a unit $v$ in $\Sigma\left(W_{L}\right)^{N_{L}}$ such that $\ell_{v}: e_{L}^{\sigma_{L}} \mathbb{C} W_{L} \rightarrow$ $e_{L}^{\tau} \mathbb{C} W_{L}$ is an isomorphism. The conclusion of the proposition is now clear, as $\mu_{v}=\ell_{x_{L}} \ell_{v} \ell_{x_{L}}^{-1}$.

The decomposition $1=\sum_{\lambda \in \Lambda} e_{\lambda}^{\sigma}$ gives a decomposition

$$
\mathbb{C} W \cong \bigoplus_{\lambda \in \Lambda} E_{\lambda}^{\sigma}
$$

of $\mathbb{C} W$ into right ideals. It is shown in [4, $\S 7]$ that $\operatorname{dim} E_{\lambda}^{\sigma}=\left|\operatorname{sh}^{-1}(\lambda)\right|$, the number of elements in $W$ with shape $\lambda$. In the next lemma we compute $\left|\operatorname{sh}^{-1}(\lambda)\right|$ in terms of cuspidal elements in a parabolic subgroup of $W$ with shape $\lambda$.

Lemma 4.5. Suppose that $\lambda$ is a shape in $\Lambda, X$ is a subspace in $\lambda$, and $C$ is a conjugacy class in $W$ with shape $\lambda$. Then

(a) $|C|=\left|W: N_{W}\left(W_{X}\right)\right|\left|C \cap W_{X}\right|$ and

(b) $\left|\operatorname{sh}^{-1}(\lambda)\right|=\left|W: N_{W}\left(W_{X}\right)\right|\left|\operatorname{sh}^{-1}(\lambda) \cap W_{X}\right|$.

Proof. Notice that $C \cap W_{X}$ is a cuspidal conjugacy class in $W_{X}$. Thus, it follows from (1) and (2) in $\$ 2.4$ that $\left|N_{W}\left(W_{X}\right): Z_{W}(c)\right|=\left|W_{X}: Z_{W_{X}}(c)\right|$ for $c$ in $C \cap W_{X}$. Therefore

$$
|C|=\left|W: N_{W}\left(W_{X}\right)\right|\left|N_{W}\left(W_{X}\right): Z_{W}(c)\right|=\left|W: N_{W}\left(W_{X}\right)\right|\left|C \cap W_{X}\right| .
$$

This proves (a). Statement (b) follows from (a) and the observation that $\operatorname{sh}^{-1}(\lambda)$ is the union of those conjugacy classes in $W$ whose intersection with $W_{X}$ is a cuspidal conjugacy class in $W_{X}$.

Corollary 4.6. Suppose $\lambda$ is in $\Lambda$, $J$ is in $S_{\lambda}, \sigma: 2^{S} \rightarrow \mathbb{R}_{>0}$, and $\tau: 2^{J} \rightarrow \mathbb{R}_{>0}$. Then

(a) $\operatorname{dim} A_{X_{J}}=\operatorname{dim} e_{J}^{\sigma} \mathbb{C} W_{J}=\operatorname{dim} e_{J}^{\tau} \mathbb{C} W_{J}=\left|\operatorname{sh}^{-1}(\lambda) \cap W_{J}\right|$ and

(b) $\operatorname{dim} A_{\lambda}=\operatorname{dim} E_{\lambda}^{\sigma}=\left|\operatorname{sh}^{-1}(\lambda)\right|$. 
Proof. It is clear that $\left\{w \in W \mid \operatorname{Fix}(w)=X_{J}\right\}=\operatorname{sh}^{-1}(\lambda) \cap W_{J}$ is the set of all cuspidal elements in $W_{J}$, and it is shown in [12, Proposition 2.4] that $\operatorname{dim} A_{X_{J}}=$ $\left|\left\{w \in W \mid \operatorname{Fix}(w)=X_{J}\right\}\right|$. Therefore, $\operatorname{dim} A_{X_{J}}=\left|\operatorname{sh}^{-1}(\lambda) \cap W_{J}\right|$, and so it follows from Lemma 4.5 (b) that $\operatorname{dim} A_{\lambda}=\left|\mathrm{sh}^{-1}(\lambda)\right|$. It is shown in [4, Theorem 7.15] that $\operatorname{dim} E_{\lambda}^{\sigma}=\left|\operatorname{sh}^{-1}(\lambda)\right|$. This proves (b).

It remains to show that $\operatorname{dim} e_{J}^{\sigma} \mathbb{C} W_{J}=\operatorname{dim} e_{J}^{\tau} \mathbb{C} W_{J}=\left|\operatorname{sh}^{-1}(\lambda) \cap W_{J}\right|$. Using Lemma 3.5(b), Lemma 3.4, Proposition 4.2, and (b) applied to the shape $\{J\}$ of $W_{J}$, we have

$$
\operatorname{dim} e_{J}^{\sigma} \mathbb{C} W_{J}=\operatorname{dim} x_{J} e_{J}^{\sigma_{J}} \mathbb{C} W_{J}=\operatorname{dim} e_{J}^{\sigma_{J}} \mathbb{C} W_{J}=\operatorname{dim} e_{J}^{\tau} \mathbb{C} W_{J}=\left|\operatorname{sh}^{-1}(\lambda) \cap W_{J}\right|,
$$

as desired.

The next proposition and its corollary are the main results in this section.

Proposition 4.7. Suppose that $\sigma: 2^{S} \rightarrow \mathbb{R}_{>0}, \lambda$ is in $\Lambda, X$ is in $\lambda$, and $L$ is in $S_{\lambda}$.

(a) $N_{W}\left(W_{L}\right)$ acts on $e_{L}^{\sigma} \mathbb{C} W_{L}$ by right multiplication and

$$
E_{\lambda}^{\sigma} \cong \operatorname{Ind}_{N_{W}\left(W_{L}\right)}^{W}\left(e_{L}^{\sigma} \mathbb{C} W_{L}\right) .
$$

(b) $N_{W}\left(W_{X}\right)$ acts on $A_{X}$ and

$$
A_{\lambda} \cong \operatorname{Ind}_{N_{W}\left(W_{X}\right)}^{W}\left(A_{X}\right) \text {. }
$$

Proof. Statement (b) is proved in [26, §2]. We prove (a).

It was shown in Proposition 4.4 that $e_{L}^{\sigma} \mathbb{C} W_{L}$ is stable under right multiplication by $N_{W}\left(W_{L}\right)$. By Lemma 4.1, $E_{\lambda}^{\sigma}=e_{L}^{\sigma} \mathbb{C} W$. Therefore, to prove that $E_{\lambda}^{\sigma} \cong \operatorname{Ind}_{N_{W}\left(W_{L}\right)}^{W}\left(e_{L}^{\sigma} \mathbb{C} W_{L}\right)$ it is enough to show that the multiplication map $e_{L}^{\sigma} \mathbb{C} W_{L} \otimes_{\mathbb{C} N_{W}\left(W_{L}\right)} \mathbb{C} W \rightarrow E_{\lambda}^{\sigma}$ is a bijection. This map is obviously a surjection. Using Lemma 4.5] and Corollary 4.6] we have

$$
\begin{aligned}
\operatorname{dim} E_{\lambda}^{\sigma} & =\left|\operatorname{sh}^{-1}(\lambda)\right| \\
& =\left|W: N_{W}\left(W_{L}\right)\right|\left|\operatorname{sh}^{-1}(\lambda) \cap W_{L}\right| \\
& =\left|W: N_{W}\left(W_{L}\right)\right| \operatorname{dim} e_{L}^{\sigma} \mathbb{C} W_{L} \\
& =\operatorname{dim} e_{L}^{\sigma} \mathbb{C} W_{L} \otimes_{\mathbb{C} N_{W}\left(W_{L}\right)} \mathbb{C} W
\end{aligned}
$$

and so the multiplication map is indeed a bijection.

We saw in Proposition 4.4 that $e_{L}^{\sigma} \mathbb{C} W_{L}, e_{L}^{\sigma_{L}} \mathbb{C} W_{L}, e_{L}^{\tau} \mathbb{C} W_{L}$, and $x_{L} e_{L}^{\tau} \mathbb{C} W_{L}$ all afford equivalent representations of $N_{W}\left(W_{L}\right)$ when $\tau: 2^{L} \rightarrow \mathbb{R}_{>0}$ is chosen only subject to the restriction that it is constant on $N_{L}$-orbits. Thus, Proposition 4.7(a) implies the following corollary.

Corollary 4.8. Suppose $\lambda$ is in $\Lambda, L$ is in $S_{\lambda}, \sigma: 2^{S} \rightarrow \mathbb{R}_{>0}$, and $\tau: 2^{L} \rightarrow \mathbb{R}_{>0}$ is constant on $N_{L}$-orbits. Then

$$
E_{\lambda}^{\sigma} \cong \operatorname{Ind}_{N_{W}\left(W_{L}\right)}^{W}\left(e_{L}^{\sigma_{L}} \mathbb{C} W_{L}\right) \cong \operatorname{Ind}_{N_{W}\left(W_{L}\right)}^{W}\left(e_{L}^{\tau} \mathbb{C} W_{L}\right) \cong \operatorname{Ind}_{N_{W}\left(W_{L}\right)}^{W}\left(x_{L} e_{L}^{\tau} \mathbb{C} W_{L}\right) .
$$

In particular, if $\sigma(I)=1$ and $\tau(J)=1$ for all $I \subseteq S$ and $J \subseteq L$, then

$$
E_{\lambda} \cong \operatorname{Ind}_{N_{W}\left(W_{L}\right)}^{W}\left(e_{L}^{L} \mathbb{C} W_{L}\right) .
$$




\section{Symmetric Groups: $\lambda=(n)$}

In this section and the next we prove Conjecture 2.1 for symmetric groups. In these two sections we take $W$ to be the symmetric group on $n$ letters with $n \geq 2$ and we identify $W$ with the subgroup of $\mathrm{GL}_{n}(\mathbb{C})$ that acts on the basis $\left\{v_{1}, v_{2}, \ldots, v_{n}\right\}$ as permutations. Here, $v_{i}$ is the column vector whose $j^{\text {th }}$ entry is 0 for $j \neq i$ and 1 for $j=i$. For $1 \leq i \leq n-1$ let $s_{i}$ denote the matrix in $W$ that interchanges $v_{i}$ and $v_{i+1}$ and fixes $v_{j}$ for $j \neq i, i+1$. Then $S=\left\{s_{1}, s_{2}, \ldots, s_{n-1}\right\}$ is a Coxeter generating set for $W$.

By a partition of $n$ we mean a non-increasing finite sequence of positive integers whose sum is $n$. Say $\lambda=\left(\lambda_{1}, \ldots, \lambda_{p}\right)$ is a partition of $n$. Then $\lambda_{1} \geq \cdots \geq \lambda_{p}>0$ and $\sum_{k=1}^{p} \lambda_{k}=n$. The integers $\lambda_{i}$ are called the parts of $\lambda$.

It is well known that for $W=S_{n}$ we may identify $\Lambda$ with the set of partitions of $n$. We make this identification precise as follows. Suppose that $\lambda$ is a partition of $n$ with $p$ parts. Define partial sums $\tau_{i}$ for $i=0,1, \ldots, p$ by $\tau_{0}=0$ and $\tau_{i}=\lambda_{1}+\cdots+\lambda_{i}$ for $1 \leq i \leq p$. Define

$$
I_{\lambda}=S \backslash\left\{s_{\tau_{1}}, s_{\tau_{2}}, \ldots, s_{\tau_{p-1}}\right\} \quad \text { and } \quad W_{\lambda}=\left\langle I_{\lambda}\right\rangle .
$$

Then $W_{\lambda}$ is isomorphic to the product of symmetric groups $S_{\lambda_{1}} \times \cdots \times S_{\lambda_{p}}$, where the factor $S_{\lambda_{i}}$ acts on the subset $\left\{v_{\tau_{i-1}+1}, v_{\tau_{i-1}+2}, \ldots, v_{\tau_{i}}\right\}$ of $\left\{v_{1}, v_{2}, \ldots, v_{n}\right\}$. Next, define

$$
X_{\lambda}=\operatorname{Fix}\left(W_{\lambda}\right) .
$$

Then $X_{\lambda}$ is in $L(\mathcal{A})$ and $W_{X_{\lambda}}=W_{\lambda}$. We have seen in Proposition 4.7 that

$$
E_{\lambda} \cong \operatorname{Ind}_{N_{W}\left(W_{\lambda}\right)}^{W}\left(e_{I_{\lambda}} \mathbb{C} W_{\lambda}\right) \text { and } A_{\lambda} \cong \operatorname{Ind}_{N_{W}\left(W_{\lambda}\right)}^{W}\left(A_{X_{\lambda}}\right) .
$$

It is well known and straightforward to check that $\left\{X_{\lambda} \mid \lambda\right.$ is a partition of $\left.n\right\}$ is a complete set of orbit representatives for the action of $W$ on $L(\mathcal{A})$ and that $\left\{I_{\lambda} \mid\right.$ $\lambda$ is a partition of $n\}$ is a complete set of representatives for the $\sim$-equivalence classes of subsets of $S$.

Notice that in the extreme case when all parts of $\lambda$ are equal to 1 we have $I_{\lambda}=\emptyset$ and $W_{\lambda}=W_{\emptyset}=\{1\}$. At the other extreme, when $\lambda=(n)$, we have $I_{\lambda}=S$ and $W_{\lambda}=W_{S}=W$. We first prove Conjecture 2.1 when $\lambda=(n)$.

For the rest of this section we take $\lambda=(n)$. Then $W_{\lambda}=N_{W}\left(W_{\lambda}\right)=W$, and so $E_{(n)}=e_{I_{(n)}} \mathbb{C} W_{(n)}$ and $A_{(n)}=A_{X_{(n)}}$. Moreover, $A_{X_{(n)}}=A^{n-1}$ is the top, non-zero graded piece of $A$. To simplify the notation, we denote $A_{(n)}, E_{(n)}$, and $e_{I_{(n)}}$ by $A_{n}$, $E_{n}$, and $e_{n}$, respectively.

Define $c_{1}=1$ in $W$ and for $1<i \leq n$ define $c_{i}=s_{i-1} \cdots s_{2} s_{1}$ so that $c_{i}$ acts on the basis $\left\{v_{1}, v_{2}, \ldots, v_{n}\right\}$ as an $i$-cycle. Also, set $c=c_{n}$. Then:

- $c$ is a cuspidal element in $W$,

- the set of cuspidal elements in $W$ is precisely the conjugacy class of $c$, and

- $Z_{W}(c)=\langle c\rangle$ is the cyclic group of order $n$ generated by $c$.

Set $\zeta=e^{2 \pi i / n}$ in $\mathbb{C}$ and define $\varphi: Z_{W}(c) \rightarrow \mathbb{C}$ by $\varphi\left(c^{-1}\right)=\zeta$. The elements we have denoted by $c_{i}$ are denoted by $c_{i}^{-1}$ by Lehrer and Solomon [26]. However, the character $\varphi$ of $Z_{W}(c)$ is the same as in [26].

Theorem 5.1. With the preceding notation we have that

(a) the character of $W$ on $E_{n}$ is $\operatorname{Ind}_{Z_{W}(c)}^{W}(\varphi)$ and

(b) the character of $W$ on $A_{n}$ is $\in \operatorname{Ind}_{Z_{W}(c)}^{W}(\varphi)$. 
Statement (b) has been proven by Stanley [36, Theorem 7.2] and by Lehrer and Solomon [26, Theorem 3.9]. As mentioned in the introduction, a proof of (a) may be extracted from classical results about the representation of $S_{n}$ on the free Lie algebra on $n$ letters. In contrast, our proof below that the character of $W$ on $E_{n}$ is $\operatorname{Ind}_{Z_{W}(c)}^{W}(\varphi)$ follows the Lehrer-Solomon argument and demonstrates a parallelism between the group algebra and the Orlik-Solomon algebra that we expect will apply in some form to all finite Coxeter groups. In addition, our argument is valid not only for $E_{n}$ and $e_{n}$, but more generally for $E_{(n)}^{\sigma}$ and $e_{(n)}^{\sigma}$ for any function $\sigma: 2^{S} \rightarrow \mathbb{R}_{>0}$.

To emphasize and differentiate the parallel arguments, we use the convention that the superscript + denotes quantities associated with $E_{n}$ and the superscript - denotes quantities associated with $A_{n}$. Notice that with the notation of 92 we have $X_{c}=\{0\}$, and so $\alpha_{c}=\left.\operatorname{det}\right|_{X_{c}}$ is the trivial character.

Suppose $t$ is an indeterminate. For $0 \leq k \leq n$, define elements $b^{+}(n, k)$ and $b^{-}(n, k)$ in $\mathbb{C} W$ by

$$
\left(1-c_{1} t\right)\left(1-c_{2} t\right) \cdots\left(1-c_{n} t\right)=\sum_{k=0}^{n} b^{+}(n, k) t^{k}
$$

and

$$
\left(1+c_{n} t\right)\left(1-c_{n-1} t\right) \cdots\left(1+(-1)^{n-1} c_{1} t\right)=\sum_{k=0}^{n} b^{-}(n, k) t^{k}
$$

respectively (the $k^{\text {th }}$ factor in the product on the left-hand side of the last equation is $\left.\left(1+(-1)^{k-1} c_{n-k+1} t\right)\right)$.

Set $W_{n-1}=\left\langle s_{1}, s_{2}, \ldots, s_{n-2}\right\rangle$. Then $W_{n-1} \cong S_{n-1}$. The analog of the idempotent $e_{n}$ in $E_{n}$ is the basis element $a_{n}=a_{s_{1}} a_{s_{2}} \cdots a_{s_{n-1}}$ in $A_{n}=A^{n-1}$. Lehrer and Solomon [26, §3] prove the following statements:

(i) $A_{n}=\mathbb{C} W a_{n}$.

(ii) $c^{-k} a_{n}=b^{-}(n-1, k) a_{n}$ for $0 \leq k \leq n-1$. In particular, $A_{n}=\mathbb{C} W_{n-1} a_{n}$.

(iii) Consider the homomorphism of left $\mathbb{C} W$-modules from $\mathbb{C} W$ to $A_{n}$ given by right multiplication by $a_{n}$. The kernel of this mapping is the left $\mathbb{C} W_{n-1^{-}}$ module generated by $\left\{c^{-k}-b^{-}(n-1, k) \mid 1 \leq k \leq n-1\right\}$.

(iv) $\left\{w a_{n} \mid w \in W_{n-1}\right\}$ is a $\mathbb{C}$-basis of $A_{n}$, and $A_{n}$ is the left regular $\mathbb{C} W_{n-1^{-}}$ module.

Next we show that the analogous statements hold with $A_{n}$ replaced by $E_{n}$ and $b^{-}(n, k)$ replaced by $b^{+}(n, k)$.

For $k=1,2, \ldots, n-1$, define $x_{k}=x_{S \backslash\left\{s_{k}\right\}}$ and $w_{k}=c_{1} c_{2} \cdots c_{k}$. Then $w_{k}$ is the longest element in $\left\langle s_{1}, s_{2}, \ldots, s_{k-1}\right\rangle$.

Lemma 5.2. Suppose $1 \leq k \leq n-1$. Then

$$
W^{S \backslash\left\{s_{k}\right\}} w_{k}=\left\{c_{i_{1}} \cdots c_{i_{k}} \mid 1 \leq i_{1}<\cdots<i_{k} \leq n\right\} .
$$

Proof. It suffices to show that if $1 \leq i_{1}<\cdots<i_{k} \leq n$, then $c_{i_{1}} \cdots c_{i_{k}} w_{k}$ is in $W^{S \backslash\left\{s_{k}\right\}}$. For this, we consider elements in $W$ as acting on $\{1, \ldots, n\}$. That is, we identify the vector $v_{j}$ with $j$ for $1 \leq j \leq n$. Then

$$
W^{S \backslash\left\{s_{k}\right\}}=\{w \in W \mid w(j)<w(j+1) \forall j \in\{1, \ldots, n-1\} \backslash\{k\}\}
$$

and

$$
w_{k}(j)= \begin{cases}k+1-j, & 1 \leq j \leq k \\ j, & k+1 \leq j \leq n\end{cases}
$$


Fix $i_{1}, \ldots, i_{k}$ with $1 \leq i_{1}<\cdots<i_{k} \leq n$ and set $w=c_{i_{1}} \cdots c_{i_{k}} w_{k}$. If $1 \leq j \leq k$, then $w(j)=i_{j}<i_{j+1}=w(j+1)$. If $j \geq i_{k}$, then $w(j) \leq j<j+1=w(j+1)$. Suppose that $k<j<i_{k}$. Choose $r$ minimal such that

$$
j+1 \leq i_{k}, j+1-1 \leq i_{k-1}, \ldots, j+1-r \leq i_{k-r}, \text { and } j+1-r-1>i_{k-r-1} .
$$

Then $w(j) \leq j-r-1<j-r=j+1-r-1=w(j+1)$.

Corollary 5.3. For $1 \leq k \leq n-1$, we have $b^{+}(n, k)=(-1)^{k} x_{k} w_{k}$.

Proof. Using the definition and Lemma 5.2 we have

$$
b^{+}(n, k)=(-1)^{k} \sum_{1 \leq i_{1}<\cdots<i_{k} \leq n} c_{i_{1}} \cdots c_{i_{k}}=(-1)^{k} x_{k} w_{k} .
$$

Proposition 5.4. The following analogs of (i)-(iv) above hold:

(a) $E_{n}=e_{n} \mathbb{C} W$.

(b) $e_{n} c^{k}=e_{n} b^{+}(n-1, k)$ for $0 \leq k \leq n-1$. In particular, $E_{n}=e_{n} \mathbb{C} W_{n-1}$.

(c) Consider the endomorphism of $\mathbb{C} W$ considered as a right $\mathbb{C} W$-module given by left multiplication by $e_{n}$. The kernel of this mapping is the free, right $\mathbb{C} W_{n-1}$-module with basis $\left\{c^{k}-b^{+}(n-1, k) \mid 1 \leq k \leq n-1\right\}$.

(d) $\left\{e_{n} w \mid w \in W_{n-1}\right\}$ is a $\mathbb{C}$-basis of $E_{n}$ and $E_{n}$ is the right regular $\mathbb{C} W_{n-1}$ module.

Proof. The first statement follows immediately from the definitions.

We prove (b) by recursion. It is clear that $e_{n} c^{k}=e_{n} b^{+}(n-1, k)$ for $k=0$, since $b^{+}(n-1,0)=1=c^{0}$. Suppose $e_{n} c^{k-1}=e_{n} b^{+}(n-1, k-1)$. It follows from [4, Theorem 7.8] that $e_{n} x_{J}=0$ unless $J=S$. Thus, it follows from Corollary 5.3 that $e_{n} b^{+}(n, k)=(-1)^{k} e_{n} x_{k} w_{k}=0$ for $1 \leq k \leq n-1$. On the other hand, it follows from the definition that

$$
\sum_{k=0}^{n} b^{+}(n, k) t^{k}=\left(\sum_{k=0}^{n-1} b^{+}(n-1, k) t^{k}\right)\left(1-c_{n} t\right),
$$

and hence $b^{+}(n, k)=b^{+}(n-1, k)-b^{+}(n-1, k-1) c$ for $1 \leq k \leq n-1$. Therefore,

$$
e_{n} c^{k}=e_{n} c^{k-1} c=e_{n} b^{+}(n-1, k-1) c=e_{n} b^{+}(n-1, k) .
$$

Next, consider the endomorphism of $\mathbb{C} W$ given by $x \mapsto e_{n} x$. Let $K$ denote the kernel of this mapping and let $K_{1}$ denote the $\mathbb{C} W_{n-1}$-submodule of $\mathbb{C} W$ generated by $\left\{c^{k}-b^{+}(n-1, k) \mid 1 \leq k \leq n-1\right\}$. It follows from (b) that $K_{1} \subseteq K$. Moreover, $\left\{c^{k}-b^{+}(n-1, k) \mid 1 \leq k \leq n-1\right\}$ is a $\mathbb{C} W_{n-1}$-basis of $K_{1}$ because the cyclic subgroup generated by $c$ is a left transversal of $W_{n-1}$ in $W$. Therefore, $\operatorname{dim}_{\mathbb{C}} K_{1}=(n-1)(n-1)$ !. However,

$$
\operatorname{dim} K=\operatorname{dim} \mathbb{C} W-\operatorname{dim} E_{n}=n !-\left|W: Z_{W}(c)\right|=(n-1)(n-1) !=\operatorname{dim} K_{1} .
$$

Therefore, $K_{1}=K$. This proves (c).

Because $b^{+}(n-1, k)$ is in $\mathbb{C} W_{n-1}$ for $1 \leq k \leq n-1$, it follows from (b) that the image of the mapping $x \mapsto e_{n} x$ is $e_{n} \mathbb{C} W_{n-1}$. Therefore, $E_{n}=e_{n} \mathbb{C} W_{n-1}$. Since $\operatorname{dim} E_{n}=(n-1)$ !, it follows that $\left\{e_{n} w \mid w \in W_{n-1}\right\}$ is a $\mathbb{C}$-basis of $E_{n}$. This proves (d). 
Finally, define idempotents $f^{+}$and $f^{-}$in $\mathbb{C} Z_{W}(c)$ by

$$
f^{+}=\frac{1}{n} \sum_{k=0}^{n-1} \varphi\left(c^{k}\right) c^{-k}=\frac{1}{n} \sum_{k=0}^{n-1} \zeta^{-k} c^{-k}
$$

and

$$
f^{-}=\frac{1}{n} \sum_{k=0}^{n-1} \epsilon(c)^{k} \varphi\left(c^{k}\right) c^{-k}=\frac{1}{n} \sum_{k=0}^{n-1} \epsilon(c)^{k} \zeta^{-k} c^{-k} .
$$

Obviously, the lines $\mathbb{C} f^{+}$and $\mathbb{C} f^{-}$in $\mathbb{C} W$ are stable under left and right multiplication by $Z_{W}(c)$ and afford the characters $\varphi$ and $\epsilon \varphi$ of $Z_{W}(c)$, respectively. Moreover, $\operatorname{Ind}_{Z_{W}(c)}^{W}(\varphi)$ is afforded by the right $\mathbb{C} W$-module $f^{+} \mathbb{C} W$, and $\epsilon \operatorname{Ind}_{Z_{W}(c)}^{W}(\varphi)=$ $\operatorname{Ind}_{Z_{W}(c)}^{W}(\epsilon \varphi)$ is afforded by the left $\mathbb{C} W$-module $\mathbb{C} W f^{-}$. Thus, to prove Theorem 5.1 it is enough to find $\mathbb{C} W$-isomorphisms $E_{n} \cong f^{+} \mathbb{C} W$ and $A_{n} \cong \mathbb{C} W f^{-}$.

Lemma 5.5. The idempotent $f^{+}$acts invertibly by right multiplication on $e_{n}$, and the idempotent $f^{-}$acts invertibly by left multiplication on $a_{n}$.

Proof. Lehrer and Solomon [26, §3] show that $f^{-}$acts invertibly on $a_{n}$. Their argument is easily modified to show that $f^{+}$acts invertibly by right multiplication on $e_{n}$ as follows.

We have $\left(1-c_{1} \zeta\right) \cdots\left(1-c_{n-1} \zeta\right)=\sum_{k=0}^{n-1} b^{+}(n-1, k) \zeta^{k}$. Multiply both sides on the left by $\frac{1}{n} e_{n}$ and use Proposition 5.4(b) to get

$$
\frac{1}{n} e_{n}\left(1-\zeta c_{1}\right) \cdots\left(1-\zeta c_{n-1}\right)=\frac{1}{n} \sum_{k=0}^{n-1} \zeta^{k} e_{n} b^{+}(n-1, k)=\frac{1}{n} \sum_{k=0}^{n-1} \zeta^{k} e_{n} c^{k}=e_{n} f^{+} .
$$

If $1 \leq k \leq n-1$, then

$$
1-\zeta^{k}=1-\zeta^{k} c_{k}^{k}=\left(1-\zeta c_{k}\right)\left(1+\zeta c_{k}+\cdots+\zeta^{k-1} c_{k}^{k-1}\right) .
$$

Since $\zeta$ is a primitive $n^{\text {th }}$ root of unity, $1-\zeta^{k} \neq 0$ in $\mathbb{C}$. Thus, $1-\zeta c_{k}$ acts invertibly on $e_{n}$ for $1 \leq k \leq n-1$, and so $f^{+}$acts invertibly on $e_{n}$.

Proof of Theorem 5.1. (See [26, §3].) Consider the mapping from $f^{+} \mathbb{C} W$ to $E_{n}$ given by $x \mapsto e_{n} x$. It follows from Lemma [5.5] and the discussion preceding it that $e_{n} f^{+} \neq 0$, that $Z_{W}(c)$ acts on the line $\mathbb{C} e_{n} f^{+}$in $E_{n}$ as the character $\varphi$, and that the mapping is a surjection. Since $\operatorname{dim} f^{+} \mathbb{C} W=\left|W: Z_{W}(c)\right|=(n-1) !=\operatorname{dim} E_{n}$, the mapping is also an injection. Thus, we have an isomorphism of right $\mathbb{C} W$-modules, $E_{n} \cong f^{+} \mathbb{C} W$.

As in [26, §3], similar reasoning applies to the mapping from $\mathbb{C} W f^{-}$to $A_{n}$ given by $x \mapsto x a_{n}$ and shows that $A_{n} \cong \mathbb{C} W f^{-}$.

\section{Symmetric groups: Arbitrary $\lambda$}

In this section we consider the case of an arbitrary partition of $n$ and complete the proof of Conjecture 2.1 for symmetric groups.

Suppose $\lambda=\left(\lambda_{1}, \lambda_{2}, \ldots, \lambda_{p}\right)$ is a partition of $n$. Recall from $\$ 5$ that $I_{\lambda}=S \backslash$ $\left\{s_{\tau_{1}}, s_{\tau_{2}}, \ldots, s_{\tau_{p-1}}\right\}$ and that $W_{\lambda}=\left\langle I_{\lambda}\right\rangle$ is isomorphic to the product of symmetric groups $S_{\lambda_{1}} \times \cdots \times S_{\lambda_{p}}$, where the factor $S_{\lambda_{i}}$ acts on $\left\{v_{\tau_{i-1}+1}, v_{\tau_{i-1}+2}, \ldots, v_{\tau_{i}}\right\}$. For $1 \leq i \leq p$ define $g_{\lambda_{i}}=s_{\tau_{i}-1} \cdots s_{\tau_{i-1}+2} s_{\tau_{i-1}+1}$. Then $g_{\lambda_{i}}$ is the $\lambda_{i}$-cycle in $S_{\lambda_{i}}$ 
that corresponds to the $n$-cycle $c=c_{n}$ in $\$ 5$ Next, define $c_{\lambda}=g_{\lambda_{1}} g_{\lambda_{2}} \cdots g_{\lambda_{p}}$ and $Z_{\lambda}=Z_{W_{\lambda}}\left(c_{\lambda}\right)$. Then

- $c_{\lambda}$ is a cuspidal element in $W_{\lambda}$,

- the set of cuspidal elements in $W_{\lambda}$ is precisely the conjugacy class of $c_{\lambda}$, and

- $Z_{\lambda} \cong\left\langle g_{\lambda_{1}}\right\rangle \times\left\langle g_{\lambda_{2}}\right\rangle \times \cdots \times\left\langle g_{\lambda_{p}}\right\rangle$.

Notice that $\left\{c_{\lambda} \mid \lambda\right.$ is a partition of $\left.n\right\}$ is a complete set of conjugacy class representatives in $W$.

With $\lambda$ as above, for $1 \leq i \leq p$, define $\varphi_{\lambda_{i}}$ to be the character of $\left\langle g_{\lambda_{i}}\right\rangle$ with $\varphi_{\lambda_{i}}\left(g_{\lambda_{i}}^{-1}\right)=e^{2 \pi i / \lambda_{i}}$. Then $\varphi_{\lambda_{i}}$ is the analog of the character $\varphi$ in $\$ 5$ for the factor $S_{\lambda_{i}}$ of $W_{\lambda}$. Next, define the character $\varphi_{\lambda}$ of $Z_{\lambda} \cong\left\langle g_{\lambda_{1}}\right\rangle \times\left\langle g_{\lambda_{2}}\right\rangle \times \cdots \times\left\langle g_{\lambda_{p}}\right\rangle$ to be

$$
\varphi_{\lambda}=\varphi_{\lambda_{1}} \otimes \cdots \otimes \varphi_{\lambda_{p}} .
$$

Note that this notation is not consistent with that of Lehrer and Solomon; our character $\varphi_{\lambda}$ corresponds to the character $\varphi_{\lambda} \epsilon$ in [26]. Applying the special case $\lambda=(n)$ considered in $\$ 5$ to each factor $S_{\lambda_{i}}$ of $W_{\lambda}$, for $1 \leq i \leq p$ define

$$
f_{\lambda_{i}}^{+}=\frac{1}{\lambda_{i}} \sum_{k=0}^{\lambda_{i}-1} \varphi_{\lambda_{i}}\left(g_{\lambda_{i}}^{k}\right) g_{\lambda_{i}}^{-k} \quad \text { and } \quad f_{\lambda_{i}}^{-}=\frac{1}{\lambda_{i}} \sum_{k=0}^{\lambda_{i}-1} \epsilon\left(g_{\lambda_{i}}^{k}\right) \varphi\left(g_{\lambda_{i}}^{k}\right) g_{\lambda_{i}}^{-k} .
$$

Finally, define idempotents $f_{\lambda}^{+}$and $f_{\lambda}^{-}$in $\mathbb{C} Z_{\lambda}$ by

$$
f_{\lambda}^{+}=f_{\lambda_{1}}^{+} f_{\lambda_{2}}^{+} \cdots f_{\lambda_{p}}^{+} \quad \text { and } \quad f_{\lambda}^{-}=f_{\lambda_{1}}^{-} f_{\lambda_{2}}^{-} \cdots f_{\lambda_{p}}^{-} .
$$

Obviously, the lines $\mathbb{C} f_{\lambda}^{+}$and $\mathbb{C} f_{\lambda}^{-}$in $\mathbb{C} W$ are stable under left and right multiplication by $Z_{\lambda}$ and afford the characters $\varphi_{\lambda}$ and $\epsilon \varphi_{\lambda}$ of $Z_{\lambda}$, respectively.

Now consider the canonical complement $N_{X_{\lambda}}$ of $W_{\lambda}$ in $N_{W}\left(W_{\lambda}\right)$. Set $N_{\lambda}=N_{X_{\lambda}}$. If $\lambda$ has $m_{j}$ parts equal to $j$, then $N_{\lambda}$ is isomorphic to the product of symmetric groups $\prod_{j} S_{m_{j}}$ (see [20] or [26]). In particular, $N_{\lambda}$ has one Coxeter generator, say $r_{i}$, for each $i$ such that $\lambda_{i}=\lambda_{i+1}$. The generator $r_{i}$ acts on the set $\left\{v_{1}, v_{2}, \ldots, v_{n}\right\}$ by interchanging $v_{\tau_{i-1}+j}$ and $v_{\tau_{i}+j}$ for $1 \leq j \leq \lambda_{i}$, and fixing $v_{k}$ for $k \leq \tau_{i-1}$ and $k>\tau_{i+1}$.

It is well known and easy to check $([26,23])$ that $N_{\lambda} \subseteq Z_{W}\left(c_{\lambda}\right)$, and so $Z_{W}\left(c_{\lambda}\right) \cong Z_{\lambda} \rtimes N_{\lambda}$.

Lemma 6.1. The subgroup $N_{\lambda}$ of $Z_{W}\left(c_{\lambda}\right)$ stabilizes the characters $\varphi_{\lambda}$ and $\epsilon \varphi_{\lambda}$ of $Z_{\lambda}$ and centralizes the idempotents $f_{\lambda}^{+}$and $f_{\lambda}^{-}$. In particular, $\varphi_{\lambda}$ extends to a character, also denoted by $\varphi_{\lambda}$, of $Z_{W}\left(c_{\lambda}\right)$, with $\varphi_{\lambda}(n z)=\varphi_{\lambda}(z)$ for $n$ in $N_{\lambda}$ and $z$ in $Z_{\lambda}$.

Proof. Suppose that $i$ is such that $\lambda_{i}=\lambda_{i+1}$ and consider the generator $r_{i}$ of $N_{\lambda}$. Then $r_{i}$ is an involution and it follows from the description of the action of $r_{i}$ on the basis $\left\{v_{1}, \ldots, v_{n}\right\}$ of $V$ that

$$
r_{i} g_{\lambda_{j}} r_{i}^{-1}=r_{i} g_{\lambda_{j}} r_{i}= \begin{cases}g_{\lambda_{i+1}}, & j=i \\ g_{\lambda_{i}}, & j=i+1, \\ g_{\lambda_{j}}, & j \neq i, i+1 .\end{cases}
$$

Since $\varphi_{\lambda}\left(g_{\lambda_{i}}\right)=\varphi_{\lambda}\left(g_{\lambda_{i+1}}\right)$, it follows that $r_{i}$ stabilizes $\varphi_{\lambda}$ and $\epsilon \varphi_{\lambda}$.

The group $N_{\lambda}$ is generated by $\left\{r_{i} \mid \lambda_{i}=\lambda_{i+1}\right\}$, and so $N_{\lambda}$ stabilizes the characters $\varphi_{\lambda}$ and $\epsilon \varphi_{\lambda}$ of $Z_{\lambda}$. Moreover, $N_{\lambda}$ acts on $\left\{g_{\lambda_{1}}, \ldots, g_{\lambda_{p}}\right\}$ by conjugation 
as a group of permutations. Thus, it follows from the definition of $f_{\lambda_{i}}^{+}$and $f_{\lambda_{i}}^{-}$ that conjugation by $N_{\lambda}$ permutes $\left\{f_{\lambda_{1}}^{+}, \ldots, f_{\lambda_{p}}^{+}\right\}$and $\left\{f_{\lambda_{1}}^{-}, \ldots, f_{\lambda_{p}}^{-}\right\}$. Since the $f_{\lambda_{i}}^{+}$'s pairwise commute and the $f_{\lambda_{i}}^{-}$'s pairwise commute, we see that $N_{\lambda}$ centralizes both $f_{\lambda_{1}}^{+} \cdots f_{\lambda_{p}}^{+}=f_{\lambda}^{+}$and $f_{\lambda_{1}}^{-} \cdots f_{\lambda_{p}}^{-}=f_{\lambda}^{-}$.

Set $\alpha_{\lambda}=\alpha_{X_{\lambda}}$. Then $\alpha_{\lambda}$ is a character of $N_{W}\left(W_{\lambda}\right)$ and $\alpha_{\lambda}\left(r_{i}\right)=-1$. Note that this notation is not consistent with that of Lehrer and Solomon; our character $\alpha_{\lambda}$ corresponds to the character $\alpha_{\lambda} \epsilon$ in [26] as $\epsilon\left(r_{i}\right)=(-1)^{\lambda_{i}}$.

Theorem 6.2. Suppose that $\lambda$ is a partition of $n$. Then the $N_{W}\left(W_{\lambda}\right)$-modules $e_{I_{\lambda}} \mathbb{C} W_{\lambda}$ and $A_{X_{\lambda}}$, and the character $\varphi_{\lambda}$ of $Z_{W}\left(c_{\lambda}\right)$, are related by

(a) the character of the right $N_{W}\left(W_{\lambda}\right)$-module $e_{I_{\lambda}} \mathbb{C} W_{\lambda}$ is $\operatorname{Ind}_{Z_{W}\left(c_{\lambda}\right)}^{N_{W}\left(W_{\lambda}\right)}\left(\varphi_{\lambda}\right)$ and

(b) the character of the left $N_{W}\left(W_{\lambda}\right)$-module $A_{X_{\lambda}}$ is $\epsilon \alpha_{\lambda} \operatorname{Ind}_{Z_{W}\left(c_{\lambda}\right)}^{N_{W}\left(W_{\lambda}\right)}\left(\varphi_{\lambda}\right)$.

Proof. Statement (b) has been proven by Lehrer and Solomon [26, Theorem 4.4]. Their argument may be rephrased as follows. Extending the definition of the element $a_{n}$ in $A_{n}$ when $\lambda=(n)$, Lehrer and Solomon define an element $a_{\lambda}$ in $A_{X_{\lambda}}$ on which $f_{\lambda}^{-}$acts invertibly. Then:

(i) $Z_{W}\left(c_{\lambda}\right)$ acts on the line $\mathbb{C} f_{\lambda}^{-} a_{\lambda}$ in $A_{X_{\lambda}}$ via the character $\epsilon_{\lambda} \alpha_{\lambda} \varphi_{\lambda}$.

(ii) $A_{X_{\lambda}}=\mathbb{C} N_{W}\left(W_{\lambda}\right) f_{\lambda}^{-} a_{\lambda}$.

(iii) The multiplication map $\mathbb{C} N_{W}\left(W_{\lambda}\right) \otimes_{\mathbb{C} Z_{W}\left(c_{\lambda}\right)} \mathbb{C} f_{\lambda}^{-} a_{\lambda} \rightarrow A_{X_{\lambda}}$ is an isomorphism.

Therefore, $A_{X_{\lambda}} \cong \operatorname{Ind}_{Z_{W}\left(c_{\lambda}\right)}^{N_{W}\left(W_{\lambda}\right)}\left(\mathbb{C} f_{\lambda}^{-} a_{\lambda}\right)$, and hence the character of $A_{X_{\lambda}}$ is indeed $\operatorname{Ind}_{Z_{W}\left(c_{\lambda}\right)}^{N_{W}\left(W_{\lambda}\right)}\left(\epsilon_{\lambda} \alpha_{\lambda} \varphi_{\lambda}\right)$.

To prove (a) we first note that by Proposition 4.4, $e_{I_{\lambda}} \mathbb{C} W_{\lambda}$ and $e_{I_{\lambda}}^{I_{\lambda}} \mathbb{C} W_{\lambda}$ are isomorphic right $N_{W}\left(W_{\lambda}\right)$-modules, and so it suffices to prove that $e_{I_{\lambda}}^{I_{\lambda}} \mathbb{C} W_{\lambda}$ affords the character $\operatorname{Ind}_{Z_{W}\left(c_{\lambda}\right)}^{N_{W}\left(W_{\lambda}\right)}\left(\varphi_{\lambda}\right)$. We argue as for $A_{X_{\lambda}}$ with $e_{I_{\lambda}}^{I_{\lambda}}$ in place of $a_{\lambda}$.

For the rest of this proof we fix a partition $\lambda=\left(\lambda_{1}, \ldots, \lambda_{p}\right)$ of $n$. To simplify the notation, set $I=I_{\lambda}$ and $e=e_{I}^{I}$. It suffices to show that the line $\mathbb{C} e f_{\lambda}^{+}$in the right $N_{W}\left(W_{\lambda}\right)$-module $e \mathbb{C} W_{\lambda}$ satisfies properties analogous to (i), (ii), and (iii) above.

$\left(\mathrm{i}^{\prime}\right) Z_{W}\left(c_{\lambda}\right)$ acts on the line $\mathbb{C} e f_{\lambda}^{+}$via the character $\varphi_{\lambda}$ : We have seen in Lemma 3.2 that $N_{\lambda}$ centralizes $e$ and in Lemma 6.1 that $N_{\lambda}$ centralizes $f_{\lambda}^{+}$. Thus, $N_{\lambda}$ centralizes $e f_{\lambda}^{+}$and $Z_{W}\left(c_{\lambda}\right)=N_{\lambda} Z_{\lambda}$ acts on the line $\mathbb{C} e f_{\lambda}^{+}$via the character $\varphi_{\lambda}$ if $e f_{\lambda}^{+} \neq 0$.

Let $\tau: 2^{I} \rightarrow \mathbb{R}_{>0}$ be the function that takes the constant value 1 . We have $I=\coprod_{j=1}^{p} I_{j}$ where $W_{\lambda_{j}}=\left\langle I_{j}\right\rangle$, and so $e=e_{I}^{\tau}=e_{I_{1}}^{\tau} \cdots e_{I_{p}}^{\tau}$ by Proposition 3.6. Therefore,

$$
e f_{\lambda}^{+}=\left(e_{I_{1}}^{\tau} \cdots e_{I_{p}}^{\tau}\right)\left(f_{\lambda_{1}}^{+} \cdots f_{\lambda_{p}}^{+}\right)=\left(e_{I_{1}}^{\tau} f_{I_{1}}^{+}\right) \cdots\left(e_{I_{p}}^{\tau} f_{\tau_{p}}^{+}\right) .
$$

For $1 \leq j \leq p$, the idempotent $e_{I_{j}}^{\tau}$ in $\mathbb{C} W_{\lambda_{j}}$ is defined by using the partition $\left(\lambda_{j}\right)$ of $\lambda_{j}$ as in $\$ 5$, and so the idempotent $f_{\lambda_{j}}^{+}$acts as a unit on $e_{I_{j}}^{\tau}$ by Lemma 5.5. Therefore, $f_{\lambda}^{+}$acts invertibly by right multiplication on $e$, and so $e f_{\lambda}^{+} \neq 0$.

(ii') $e \mathbb{C} W_{\lambda}=e f_{\lambda}^{+} \mathbb{C} N_{W}\left(W_{\lambda}\right)$ : Because $f_{\lambda}^{+}$acts invertibly on $e$ and $N_{W}\left(W_{\lambda}\right)=$ $N_{\lambda} Z_{\lambda} W_{\lambda}$, we have

$$
e \mathbb{C} W_{\lambda}=e f_{\lambda}^{+} \mathbb{C} W_{\lambda}=e f_{\lambda}^{+} \mathbb{C} N_{\lambda} Z_{\lambda} W_{\lambda}=e f_{\lambda}^{+} \mathbb{C} N_{W}\left(W_{\lambda}\right) .
$$


(iii') The multiplication map $\mathbb{C} e f_{\lambda}^{+} \otimes_{\mathbb{C} Z_{W}\left(c_{\lambda}\right)} \mathbb{C} N_{W}\left(W_{\lambda}\right) \rightarrow e \mathbb{C} W_{\lambda}$ is an isomorphism: It follows from (ii') that the mapping is surjective. Moreover, using Corollary 4.6 we have

$$
\begin{aligned}
\operatorname{dim} e f_{\lambda}^{+} \mathbb{C} N_{W}\left(W_{\lambda}\right) & =\operatorname{dim} e \mathbb{C} W_{\lambda} \\
& =\left|W_{\lambda}: Z_{\lambda}\right| \\
& =\left|N_{W}\left(W_{\lambda}\right): Z_{W}\left(c_{\lambda}\right)\right| \\
& =\operatorname{dim} \mathbb{C} e f_{\lambda}^{+} \otimes_{\mathbb{C} Z_{W}\left(c_{\lambda}\right)} \mathbb{C} N_{W}\left(W_{\lambda}\right),
\end{aligned}
$$

and so the mapping is an isomorphism.

This completes the proof of the theorem.

The proof of Conjecture 2.1 for symmetric groups now follows from Proposition 4.7. Theorem 6.2, and transitivity of induction.

Theorem 6.3. For each partition $\lambda$ of $n$ there is a linear character $\varphi_{\lambda}$ of $Z_{W}\left(c_{\lambda}\right)$ such that

(a) the character of $E_{\lambda}$ is $\operatorname{Ind}_{Z_{W}\left(c_{\lambda}\right)}^{W}\left(\varphi_{\lambda}\right)$ and

(b) the character of $A_{\lambda}$ is $\operatorname{Ind}_{Z_{W}\left(c_{\lambda}\right)}^{W}\left(\epsilon_{\lambda} \alpha_{\lambda} \varphi_{\lambda}\right)$, where $\epsilon_{\lambda}$ denotes the restriction of $\epsilon$ to $Z_{W}\left(c_{\lambda}\right)$.

In particular,

for $0 \leq p \leq n-1$, and

$$
H^{p}\left(M_{W}\right) \cong \bigoplus_{\lambda \vdash n, \operatorname{rk}\left(c_{\lambda}\right)=p} \operatorname{Ind}_{Z_{W}\left(c_{\lambda}\right)}^{W}\left(\epsilon_{\lambda} \alpha_{\lambda} \varphi_{\lambda}\right)
$$

$$
\mathbb{C} W \cong \bigoplus_{\lambda \vdash n} \operatorname{Ind}_{Z_{W}\left(c_{\lambda}\right)}^{W}\left(\varphi_{\lambda}\right) \quad \text { and } \quad A \cong \bigoplus_{\lambda \vdash n} \operatorname{Ind}_{Z_{W}\left(c_{\lambda}\right)}^{W}\left(\epsilon_{\lambda} \alpha_{\lambda} \varphi_{\lambda}\right) .
$$

\section{Parabolic subgroups of type $A$}

In this section we return to the case when $W$ is an arbitrary finite Coxeter group and prove a relative version of Theorem 6.3 .

Suppose that $\lambda$ is in $\Lambda, c$ is in $W$ with $\operatorname{sh}(c)=\lambda$, and that all the irreducible components of $W_{c}$ are of type $A$. Without loss of generality we may assume that $W_{c}=W_{L}$ is a standard parabolic subgroup. Suppose that $L=\coprod_{i=1}^{p} L_{i}$, where $\left|L_{i}\right|=l_{i}$ and each $L_{i}$ is of type $A_{l_{i}}$. Define $n_{i}=l_{i}+1$. Then

$$
W_{L} \cong W_{L_{1}} \times \cdots \times W_{L_{p}} \cong S_{n_{1}} \times \cdots \times S_{n_{p}} .
$$

We assume that $l_{1} \geq \cdots \geq l_{p}, L_{i}=\left\{s_{i, 1}, \ldots, s_{i, l_{i}}\right\}$, and $\Delta_{L_{i}}=\left\{\alpha_{i, 1}, \ldots, \alpha_{i, l_{i}}\right\}$, where the labeling is such that $s_{i, j}$ and $s_{i, k}$ commute if $|j-k|>1$.

Define $c_{i}=s_{i, l_{i}} \cdots s_{i, 2} s_{i, 1}$ in $W_{L_{i}}$ and $c=c_{1} \cdots c_{p}$. Then $c$ is a cuspidal element in $W_{L}$ and the set of cuspidal elements in $W_{L}$ is precisely the $W_{L}$-conjugacy class of $c$. For $1 \leq i \leq p$ define $\varphi_{i}$ to be the character of $\left\langle c_{i}\right\rangle$ with $\varphi_{i}\left(c_{i}^{-1}\right)=\zeta_{n_{i}}$, where $\zeta_{n_{i}}$ is a fixed primitive $n_{i}{ }^{\text {th }}$ root of unity, and set $\varphi_{c}=\varphi_{1} \otimes \cdots \otimes \varphi_{p}$. Then $\varphi_{c}$ is a character of $Z_{W_{L}}(c) \cong\left\langle c_{1}\right\rangle \times \cdots \times\left\langle c_{p}\right\rangle$.

The rest of this section is devoted to the proof of the following theorem.

Theorem 7.1. The character $\varphi_{c}$ of $Z_{W_{L}}(c)$ extends to a character $\widetilde{\varphi}_{c}$ of $Z_{W}(c)$ such that

$$
E_{\lambda} \cong \operatorname{Ind}_{Z_{W}(c)}^{W}\left(\widetilde{\varphi}_{c}\right) \quad \text { and } \quad A_{\lambda} \cong \operatorname{Ind}_{Z_{W}(c)}^{W}\left(\epsilon_{c} \alpha_{c} \widetilde{\varphi}_{c}\right) \text {. }
$$


The proof follows the same outline as in 8 ; We find lines in $e_{L}^{L} \mathbb{C} W_{L}$ and $A_{X_{L}}$ such that the analogs of statements (i), (ii), and (iii) and $\left(\mathrm{i}^{\prime}\right),\left(\mathrm{ii}^{\prime}\right)$, and (iii') in the proof of Theorem 6.2 hold, and so

$$
e_{L}^{L} \mathbb{C} W_{L} \cong \operatorname{Ind}_{Z_{W}(c)}^{N_{W}\left(W_{L}\right)}\left(\widetilde{\varphi}_{c}\right) \quad \text { and } \quad A_{X_{L}} \cong \operatorname{Ind}_{Z_{W}(c)}^{N_{W}\left(W_{L}\right)}\left(\epsilon \alpha_{c} \widetilde{\varphi}_{c}\right) .
$$

We then apply $\operatorname{Ind}_{N_{W}\left(W_{L}\right)}^{W}$ to both sides of both equations, and the theorem follows from Proposition 4.7 by transitivity of induction. The argument in this section is complicated by the fact that the subgroup $N_{L}$ is not necessarily contained in $Z_{W}(c)$.

In case $W$ is a symmetric group, it was shown in Lemma 6.1 that $\widetilde{\varphi}_{c}$ is the trivial extension of $\varphi_{c}$. In the general case, this is no longer so. Formulas for $\widetilde{\varphi}_{c}$ are given in the proof of Lemma 7.3 below. Notice that $c$ is an involution if and only if $l_{1}=1$ for $1 \leq i \leq p$ and that in this case $\varphi_{c}$ is the sign character of $Z_{W_{L}}(c)$ and $\widetilde{\varphi}_{c}$ is the trivial extension of $\varphi_{c}$ to $Z_{W}(c)$.

Although $N_{L}$ is not necessarily contained in $Z_{W}(c)$, Konvalinka, Pfeiffer, and Röver 23] have shown that $Z_{W_{L}}(c)$ does have a complement, $N_{c}$, in $Z_{W}(c)$, and $N_{c}$ is also a complement to $W_{L}$ in $N_{W}\left(W_{L}\right)$.

By [20], the group $N_{L}$ is generated by $\left\{r_{i}, g_{j} \mid 1 \leq i \leq p-1,1 \leq j \leq p\right\}$, where $r_{i}$ and $g_{i}$ act on $W_{L}$ as follows:

- If $n_{i} \neq n_{i+1}$, then $r_{i}=1$. If $n_{i}=n_{i+1}$, then $r_{i}$ acts on $L$ by

$$
r_{i} s_{j, k} r_{i}^{-1}= \begin{cases}s_{i+1, k} & \text { if } j=i, \\ s_{i, k} & \text { if } j=i+1, \\ s_{j, k} & \text { otherwise. }\end{cases}
$$

In particular, $r_{i}$ is an involution,

$$
r_{i} c_{j} r_{i}= \begin{cases}c_{i+1} & \text { if } j=i, \\ c_{i} & \text { if } j=i+1, \\ c_{j} & \text { otherwise, }\end{cases}
$$

and $r_{i}$ is in $Z_{W}(c)$.

- Either $g_{i}=1$ or $g_{i}$ acts on $L$ by

$$
g_{i} s_{j, k} g_{i}^{-1}= \begin{cases}s_{i, n_{i}-k} & \text { if } j=i, \\ s_{j, k} & \text { if } j \neq i .\end{cases}
$$

In particular, $g_{i}$ is an involution, and if $g_{i} \neq 1$, then

$$
g_{i} c_{j} g_{i}= \begin{cases}c_{i}^{-1} & \text { if } j=i, \\ c_{j} & \text { if } j \neq i .\end{cases}
$$

Notice that if $W$ is a symmetric group, then $g_{i}=1$ for $1 \leq i \leq p$.

For $1 \leq i \leq p$, let $w_{i}$ denote the longest element in $W_{L_{i}}$ and define

$$
h_{i}= \begin{cases}1 & \text { if } g_{i}=1, \\ g_{i} w_{i} & \text { if } g_{i} \neq 1 .\end{cases}
$$

Then $h_{i}$ is in $Z_{W}\left(c_{j}\right)$ for $1 \leq i, j \leq p$. It is shown in [23] that

$$
N_{c}=\left\langle r_{i}, h_{j} \mid 1 \leq i \leq p-1,1 \leq j \leq p\right\rangle
$$

is a complement to $Z_{W_{L}}(c)$ in $Z_{W}(c)$ and to $W_{L}$ in $N_{W}\left(W_{L}\right)$. 
As in 96 define

$$
\begin{aligned}
f_{i}^{+} & =\frac{1}{n_{i}} \sum_{k=0}^{n_{i}-1} \varphi_{i}\left(c_{i}^{k}\right) c_{i}^{-k}, & f_{i}^{-} & =\frac{1}{n_{i}} \sum_{k=0}^{n_{i}-1} \epsilon\left(c_{i}^{k}\right) \varphi_{i}\left(c_{i}^{k}\right) c_{i}^{-k}, \\
f_{L}^{+} & =f_{1}^{+} f_{2}^{+} \cdots f_{p}^{+}, & f_{L}^{-} & =f_{1}^{-} f_{2}^{-} \cdots f_{p}^{-} .
\end{aligned}
$$

Then the lines $\mathbb{C} f_{L}^{+}$and $\mathbb{C} f_{L}^{-}$in $\mathbb{C} W$ are stable under left and right multiplication by $Z_{W_{L}}(c)$ and afford the characters $\varphi_{c}$ and $\epsilon \varphi_{c}$ of $Z_{W_{L}}(c)$, respectively. Because $h_{i}$ centralizes $c_{j}$ for $1 \leq i, j \leq p$, the proof of the second statement in Lemma 6.1 applies word-for-word to $N_{c}$ and proves the next lemma.

Lemma 7.2. The subgroup $N_{c}$ of $Z_{W}(c)$ centralizes the idempotents $f_{L}^{+}$and $f_{L}^{-}$in $\mathbb{C} Z_{W_{L}}(c)$.

For $1 \leq i \leq p$, define $a_{i}=a_{s_{i, 1}} \cdots a_{s_{i, l_{i}}}$. Set $a_{L}=a_{1} \cdots a_{p}$. Then $a_{L}$ is in $A_{X_{L}}$. The next lemma is the analog of statements (i) and ( $\left.\mathrm{i}^{\prime}\right)$ in the proof of Theorem 6.2 .

Lemma 7.3. The lines $\mathbb{C} e_{L}^{L} f_{L}^{+}$in $e_{L}^{L} \mathbb{C} W_{L}$ and $\mathbb{C} f_{L}^{-} a_{L}$ in $A_{X_{L}}$ are non-zero and $Z_{W}(c)$-stable. Let $\widetilde{\varphi}_{c}$ be the character of $Z_{W}(c)$ acting by right multiplication on the line $\mathbb{C} e_{L}^{L} f_{L}^{+}$. Then $\widetilde{\varphi}_{c}$ is an extension of $\varphi_{c}$, and the character of $Z_{W}(c)$ acting by left multiplication on the line $\mathbb{C} f_{L}^{-} a_{L}$ is $\epsilon \alpha_{c} \widetilde{\varphi}_{c}$.

Proof. The argument in statement $\left(\mathrm{i}^{\prime}\right)$ in 6 applies verbatim to $f_{L}^{+}$and shows that $f_{L}^{+}$acts invertibly by right multiplication on $e_{L}^{L}$, and so $e_{L}^{L} f_{L}^{+} \neq 0$. Similarly, the argument in Lehrer and Solomon [26, §3] shows that $f_{i}^{-}$acts as a unit on $a_{i}$ for $1 \leq i \leq p$. It follows that $f_{L}^{-}$acts invertibly by left multiplication on $a_{L}$, and so $f_{L}^{-} a_{L} \neq 0$. Therefore, $Z_{W_{L}}(c)$ acts on $\mathbb{C} e_{L}^{L} f_{L}^{+}$and $\mathbb{C} f_{L}^{-} a_{L}$ by $\varphi_{c}$ and $\epsilon \varphi_{c}$, respectively, and $\widetilde{\varphi}_{c}$ extends $\varphi_{c}$.

If $n_{i}=n_{i+1}$, then as in $₫ 6$ we have

$$
\left(e_{L}^{L} f_{L}^{+}\right) \cdot r_{i}=\left(e_{L}^{L} \cdot r_{i}\right) f_{L}^{+}=\left(r_{i} e_{L}^{L} r_{i}\right) f_{L}^{+}=e_{L}^{L} f_{L}^{+}
$$

and

$$
\begin{aligned}
& r_{i} \cdot f_{L}^{-} a_{L}=f_{L}^{-}\left(r_{i} \cdot a_{L}\right)=f_{L}^{-} \cdot a_{1} \cdots a_{i-1} a_{i+1} a_{i} a_{i+2} \cdots a_{p} \\
&=(-1)^{l_{i}^{2}} f_{L}^{-} \cdot a_{L}=(-1)^{l_{i}} f_{L}^{-} \cdot a_{L} .
\end{aligned}
$$

If $h_{i} \neq 1$, then

$$
e_{L}^{L} f_{L}^{+} \cdot h_{i}=\left(e_{L}^{L} \cdot g_{i} w_{i}\right) f_{L}^{+}=\left(g_{i} e_{L}^{L} g_{i} w_{i}\right) f_{L}^{+}=\left(e_{L}^{L} w_{i}\right) f_{L}^{+}=(-1)^{l_{i}} e_{L}^{L} f_{L}^{+}
$$

(the last equality follows from Lemma 3.3), and

$$
h_{i} \cdot f_{L}^{-} a_{L}=f_{L}^{-}\left(h_{i} \cdot a_{L}\right)=f_{L}^{-} a_{L}
$$

since $h_{i}$ centralizes $L$. It follows that $Z_{W}(c)$ acts on the lines $\mathbb{C} e_{L}^{L} f_{L}^{+}$and $\mathbb{C} f_{L}^{-} a_{L}$. Moreover, from (7.1) and (7.3) we see that

$$
\widetilde{\varphi}_{c}\left(r_{i}\right)=1 \quad \text { and } \quad \widetilde{\varphi}_{c}\left(h_{i}\right)=(-1)^{l_{i}} .
$$


To complete the proof we need to show that $Z_{W}(c)$ acts on the line $\mathbb{C} f_{L}^{-} a_{L}$ as $\epsilon \alpha_{c} \widetilde{\varphi}_{c}$

For $w$ in $Z_{W_{L}}(c)$ we have $\alpha_{c}(w)=1$ and $\widetilde{\varphi}_{c}(w)=\varphi_{c}(w)$. Hence

$$
w \cdot f_{L}^{-} a_{L}=\epsilon(w) \varphi_{c}(w) \cdot f_{L}^{-} a_{L}=\left(\epsilon \alpha_{c} \widetilde{\varphi}_{c}\right)(w) f_{L}^{-} a_{L} .
$$

For $n$ in $N_{L}$ it is shown in [13, Lemma 2.1] that $\epsilon(n) \alpha_{c}(n)$ is the sign of the permutation of $L$ induced by conjugation by $n$.

Suppose next that $1 \leq i \leq p-1$ and $n_{i}=n_{i+1}$. Then the sign of the permutation of $L$ induced by conjugation by $r_{i}$ is $(-1)^{l_{i}}$, and so $\epsilon\left(r_{i}\right) \alpha_{c}\left(r_{i}\right)=(-1)^{l_{i}}$. Therefore, using (7.2) we see that

$$
r_{i} \cdot f_{L}^{-} a_{L}=(-1)^{l_{i}} f_{L}^{-} a_{L}=\left(\epsilon \alpha_{c} \widetilde{\varphi}_{c}\right)\left(r_{i}\right) f_{L}^{-} a_{L} .
$$

Finally, suppose that $1 \leq i \leq p$ and that $g_{i} \neq 1$. Then the sign of the permutation of $L$ induced by conjugation by $g_{i}$ is 1 if $l_{i} \equiv 0,1(\bmod 4)$ and -1 if $l_{i} \equiv 2,3$ $(\bmod 4)$. Thus, $\epsilon\left(g_{i}\right) \alpha_{c}\left(g_{i}\right)=1$ if $l_{i} \equiv 0,1(\bmod 4)$ and $\epsilon\left(g_{i}\right) \alpha_{c}\left(g_{i}\right)=-1$ if $l_{i} \equiv 2,3$ $(\bmod 4)$. Since $\ell\left(w_{i}\right)=\left(\begin{array}{c}l_{i}+1 \\ 2\end{array}\right)$, it follows that $\epsilon\left(w_{i}\right)=1$ if $l_{i} \equiv 0,3(\bmod 4)$ and $\epsilon\left(w_{i}\right)=-1$ if $l_{i} \equiv 1,2(\bmod 4)$. Hence $\epsilon\left(g_{i}\right) \alpha_{c}\left(g_{i}\right) \epsilon\left(w_{i}\right)=(-1)^{l_{i}}$. Because $\alpha_{c}\left(w_{i}\right)=1$ we conclude that

$$
\epsilon\left(h_{i}\right) \alpha_{c}\left(h_{i}\right)=\epsilon\left(g_{i}\right) \epsilon\left(w_{i}\right) \alpha_{c}\left(g_{i}\right) \alpha_{c}\left(w_{i}\right)=(-1)^{l_{i}}=\widetilde{\varphi}_{c}\left(h_{i}\right) .
$$

Therefore, using (7.4) we see that

$$
h_{i} \cdot f_{L}^{-} a_{L}=f_{L}^{-} a_{L}=\left(\epsilon \alpha_{c} \widetilde{\varphi}_{c}\right)\left(h_{i}\right) f_{L}^{-} a_{L} .
$$

This completes the proof of the lemma.

Because $f_{\lambda}^{+}$acts invertibly on $e_{L}^{L}, N_{c}$ acts on the line $\mathbb{C} e_{L}^{L} f_{L}^{+}$by scalars, and $N_{W}\left(W_{L}\right)=N_{c} W_{L}$, we see that

$$
e_{L}^{L} \mathbb{C} W_{L}=e_{L}^{L} f_{L}^{+} \mathbb{C} W_{L}=e_{L}^{L} f_{L}^{+} \mathbb{C} N_{c} W_{L}=e_{L}^{L} f_{L}^{+} \mathbb{C} N_{W}\left(W_{L}\right) .
$$

Lehrer and Solomon [26, Proposition 4.4(ii)] have shown that $A_{X_{L}}=\mathbb{C} W_{L} a_{L}$. Thus, because $f_{\lambda}^{-}$acts invertibly on $a_{L}, N_{c}$ acts on the line $\mathbb{C} f_{L}^{-} a_{L}$ by scalars, and $N_{W}\left(W_{L}\right)=W_{L} N_{c}$, we see that

$$
A_{X_{L}}=\mathbb{C} W_{L} a_{L}=\mathbb{C} W_{L} f_{L}^{-} a_{L}=\mathbb{C} W_{L} N_{c} f_{L}^{-} a_{L}=\mathbb{C} N_{W}\left(W_{L}\right) f_{L}^{-} a_{L} .
$$

Equations (7.5) and (7.6) are the analogs of statements (ii) and (ii') in the proof of Theorem 6.2 The dimension computation in the proof of statement (iii') now applies to show that the analogs of statements (iii) and (iii') both hold in the present situation: The multiplication maps

$\mathbb{C} e_{L}^{L} f_{L}^{+} \otimes_{\mathbb{C} Z_{W}(c)} \mathbb{C} N_{W}\left(W_{L}\right) \rightarrow e_{L}^{L} \mathbb{C} W_{L} \quad$ and $\quad \mathbb{C} N_{W}\left(W_{L}\right) \otimes_{\mathbb{C} Z_{W}(c)} \mathbb{C} f_{L}^{-} a_{L} \rightarrow A_{X_{L}}$

are isomorphisms of $\mathbb{C} N_{W}\left(W_{L}\right)$-modules.

It follows from (7.7) and Lemma 7.3 that

$$
e_{L}^{L} \mathbb{C} W_{L} \cong \operatorname{Ind}_{Z_{W}(c)}^{N_{W}\left(W_{L}\right)}\left(\widetilde{\varphi}_{c}\right) \quad \text { and } \quad A_{X_{L}} \cong \operatorname{Ind}_{Z_{W}(c)}^{N_{W}\left(W_{L}\right)}\left(\epsilon \alpha_{c} \widetilde{\varphi}_{c}\right)
$$


Apply $\operatorname{Ind}_{N_{W}\left(W_{L}\right)}^{W}$ to both sides of these last two equations and use transitivity of induction to get

$$
\operatorname{Ind}_{N_{W}\left(W_{L}\right)}^{W}\left(e_{L}^{L} \mathbb{C} W_{L}\right) \cong \operatorname{Ind}_{Z_{W}(c)}^{W}\left(\widetilde{\varphi}_{c}\right)
$$

and

$$
\operatorname{Ind}_{N_{W}\left(W_{L}\right)}^{W}\left(A_{X_{L}}\right) \cong \operatorname{Ind}_{Z_{W}(c)}^{W}\left(\epsilon \alpha_{c} \widetilde{\varphi}_{c}\right)
$$

By Proposition 4.7 and Corollary 4.8 we have

$$
E_{\lambda} \cong \operatorname{Ind}_{Z_{W}(c)}^{W}\left(\widetilde{\varphi}_{c}\right) \text { and } A_{\lambda} \cong \operatorname{Ind}_{Z_{W}(c)}^{W}\left(\epsilon \alpha_{c} \widetilde{\varphi}_{c}\right) .
$$

This completes the proof of the theorem.

\section{ACKNOWLEDGEMENTS}

The authors would like to acknowledge support from the DFG-priority programme SPP1489 "Algorithmic and Experimental Methods in Algebra, Geometry, and Number Theory". Part of the research for this paper was carried out while the authors were staying at the Mathematical Research Institute Oberwolfach supported by the "Research in Pairs" programme. The second author wishes to acknowledge support from Science Foundation Ireland.

\section{REFERENCES}

[1] Hélène Barcelo and Nantel Bergeron, The Orlik-Solomon algebra on the partition lattice and the free Lie algebra, J. Combin. Theory Ser. A 55 (1990), no. 1, 80-92, DOI 10.1016/00973165(90)90049-3. MR1070017 (91i:05119)

[2] François Bergeron and Nantel Bergeron, Orthogonal idempotents in the descent algebra of $B_{n}$ and applications, J. Pure Appl. Algebra 79 (1992), no. 2, 109-129, DOI 10.1016/00224049(92)90153-7. MR 1163285 (93f:20054)

[3] F. Bergeron, N. Bergeron, and A. M. Garsia, Idempotents for the free Lie algebra and $q$ enumeration. In Invariant theory and tableaux (Minneapolis, MN, 1988), volume 19 of IMA Vol. Math. Appl., pages 166-190. Springer, New York, 1990.

[4] F. Bergeron, N. Bergeron, R. B. Howlett, and D. E. Taylor, A decomposition of the descent algebra of a finite Coxeter group, J. Algebraic Combin. 1 (1992), no. 1, 23-44, DOI 10.1023/A:1022481230120. MR:1162640 (93g:20079)

[5] Nantel Bergeron, Hyperoctahedral operations on Hochschild homology, Adv. Math. 110 (1995), no. 2, 255-276, DOI 10.1006/aima.1995.1011. MR:1317618 (96h:16009)

[6] Marcus Bishop, J. Matthew Douglass, Götz Pfeiffer, and Gerhard Röhrle, Computations for Coxeter arrangements and Solomon's descent algebra: Groups of rank three and four, J. Symbolic Comput. 50 (2013), 139-158, DOI 10.1016/j.jsc.2012.06.001. MR2996872

[7] M. Bishop, J.M. Douglass, G. Pfeiffer, and G. Röhrle. Computations for Coxeter arrangements and Solomon's descent algebra II: Groups of rank five and six, J. Algebra 377 (2013), 320-332. MR.3008911

[8] M. Bishop, J.M. Douglass, G. Pfeiffer, and G. Röhrle, Computations for Coxeter arrangements and Solomon's descent algebra III: Groups of rank seven and eight, Arxiv: http://arxiv.org/abs/1403.6227

[9] J. Blair and G. I. Lehrer, Cohomology actions and centralisers in unitary reflection groups, Proc. London Math. Soc. (3) 83 (2001), no. 3, 582-604, DOI 10.1112/plms/83.3.582. MR:1851083 (2002g:14093)

[10] Angeline Brandt, The free Lie ring and Lie representations of the full linear group, Trans. Amer. Math. Soc. 56 (1944), 528-536. MR0011305 (6,146d)

[11] Egbert Brieskorn, Sur les groupes de tresses [d'après V. I. Arnol'd] (French), Séminaire Bourbaki, 24ème année (1971/1972), Exp. No. 401, Springer, Berlin, 1973, pp. 21-44. Lecture Notes in Math., Vol. 317. MR0422674 (54 \#10660)

[12] J. Matthew Douglass, On the cohomology of an arrangement of type $B_{l}$, J. Algebra 147 (1992), no. 2, 265-282, DOI 10.1016/0021-8693(92)90206-2. MR1161294(93d:52017) 
[13] J. Matthew Douglass, Götz Pfeiffer, and Gerhard Röhrle, An inductive approach to Coxeter arrangements and Solomon's descent algebra, J. Algebraic Combin. 35 (2012), no. 2, 215-235, DOI 10.1007/s10801-011-0301-9. MR2886288 (2012m:05431)

[14] G. Felder and A. P. Veselov, Coxeter group actions on the complement of hyperplanes and special involutions, J. Eur. Math. Soc. (JEMS) 7 (2005), no. 1, 101-116, DOI 10.4171/JEMS/23. MR2120992(2006c:20082)

[15] A. M. Garsia and C. Reutenauer, A decomposition of Solomon's descent algebra, Adv. Math. 77 (1989), no. 2, 189-262, DOI 10.1016/0001-8708(89)90020-0. MR1020585 (91c:20007)

[16] Meinolf Geck, Gerhard Hiss, Frank Lübeck, Gunter Malle, and Götz Pfeiffer, CHEVIE-a system for computing and processing generic character tables, Appl. Algebra Engrg. Comm. Comput. 7 (1996), no. 3, 175-210, DOI 10.1007/BF01190329. Computational methods in Lie theory (Essen, 1994). MR 1486215 (99m:20017)

[17] M. Geck and G. Malle, Frobenius-Schur indicators of unipotent characters and the twisted involution module, Represent. Theory 17 (2013), 180-198. MR3037782

[18] Meinolf Geck and Götz Pfeiffer, Characters of finite Coxeter groups and Iwahori-Hecke algebras, London Mathematical Society Monographs. New Series, vol. 21, The Clarendon Press Oxford University Press, New York, 2000. MR.1778802 (2002k:20017)

[19] Phil Hanlon, The action of $S_{n}$ on the components of the Hodge decomposition of Hochschild homology, Michigan Math. J. 37 (1990), no. 1, 105-124, DOI 10.1307/mmj/1029004069. MR 1042517 (91g:20013)

[20] Robert B. Howlett, Normalizers of parabolic subgroups of reflection groups, J. London Math. Soc. (2) 21 (1980), no. 1, 62-80, DOI 10.1112/jlms/s2-21.1.62. MR576184 (81g:20094)

[21] R. B. Howlett and G. I. Lehrer, Duality in the normalizer of a parabolic subgroup of a finite Coxeter group, Bull. London Math. Soc. 14 (1982), no. 2, 133-136, DOI 10.1112/blms/14.2.133. MR647196 (83e:20049)

[22] A. A. Klyachko, Lie elements in the tensor algebra, Siberian Math. J. 15 (1974), 914-921.

[23] Matjaž Konvalinka, Götz Pfeiffer, and Claas E. Röver, A note on element centralizers in finite Coxeter groups, J. Group Theory 14 (2011), no. 5, 727-745, DOI 10.1515/JGT.2010.074. MR2831968(2012j:20125)

[24] Robert E. Kottwitz, Involutions in Weyl groups, Represent. Theory 4 (2000), 1-15 (electronic), DOI 10.1090/S1088-4165-00-00050-9. MR1740177 (2000m:22014)

[25] G. I. Lehrer, On the Poincaré series associated with Coxeter group actions on complements of hyperplanes, J. London Math. Soc. (2) 36 (1987), no. 2, 275-294, DOI 10.1112/jlms/s236.2.275. MR906148 (88k:32037)

[26] G. I. Lehrer and Louis Solomon, On the action of the symmetric group on the cohomology of the complement of its reflecting hyperplanes, J. Algebra 104 (1986), no. 2, 410-424, DOI 10.1016/0021-8693(86)90225-5. MR866785 (88a:32017)

[27] G. Lusztig and D. Vogan, Hecke algebras and involutions in Weyl groups, Bull. Inst. Math. Acad. Sin. (N.S.) 7 (2012), no. 3, 323-354. MR.3051317

[28] Peter Orlik and Louis Solomon, Combinatorics and topology of complements of hyperplanes, Invent. Math. 56 (1980), no. 2, 167-189, DOI 10.1007/BF01392549. MR.558866 (81e:32015)

[29] Peter Orlik and Louis Solomon, Coxeter arrangements, Singularities, Part 2 (Arcata, Calif., 1981), Proc. Sympos. Pure Math., vol. 40, Amer. Math. Soc., Providence, RI, 1983, pp. 269291. MR:713255 (85b:32016)

[30] Peter Orlik and Hiroaki Terao, Arrangements of hyperplanes, Grundlehren der Mathematischen Wissenschaften [Fundamental Principles of Mathematical Sciences], vol. 300, SpringerVerlag, Berlin, 1992. MR1217488 (94e:52014)

[31] Christophe Reutenauer, Free Lie algebras, London Mathematical Society Monographs. New Series, vol. 7, The Clarendon Press Oxford University Press, New York, 1993. Oxford Science Publications. MR 1231799 (94j:17002)

[32] M. Schönert et al. GAP - Groups, Algorithms, and Programming - version 3 release 4, Lehrstuhl D für Mathematik, Rheinisch Westfälische Technische Hochschule, Aachen, Germany, 1997.

[33] Manfred Schocker, Über die höheren Lie-Darstellungen der symmetrischen Gruppen (German, with English summary), Bayreuth. Math. Schr. 63 (2001), 103-263. MR1867282 (2002i:20018)

[34] Louis Solomon, A decomposition of the group algebra of a finite Coxeter group, J. Algebra 9 (1968), 220-239. MR0232868 (38 \#1191) 
[35] Louis Solomon, A Mackey formula in the group ring of a Coxeter group, J. Algebra 41 (1976), no. 2, 255-264. MR0444756 (56 \#3104)

[36] Richard P. Stanley, Some aspects of groups acting on finite posets, J. Combin. Theory Ser. A 32 (1982), no. 2, 132-161, DOI 10.1016/0097-3165(82)90017-6. MR654618 (83d:06002)

[37] Robert Steinberg, Differential equations invariant under finite reflection groups, Trans. Amer. Math. Soc. 112 (1964), 392-400. MR0167535 (29 \#4807)

[38] Jacques Thévenaz, G-algebras and modular representation theory, Oxford Mathematical Monographs, The Clarendon Press Oxford University Press, New York, 1995. Oxford Science Publications. MR1365077 (96j:20017)

[39] Franz Wever, Über Invarianten in Lie'schen Ringen (German), Math. Ann. 120 (1949), 563-580. MR0029893(10,676e)

Department of Mathematics, University of North Texas, Denton, Texas 76203

E-mail address: douglass@unt.edu

$U R L:$ http://hilbert.math.unt.edu

School of Mathematics, Statistics and Applied Mathematics, National University of Ireland Galway, Galway, Ireland

E-mail address: goetz.pfeiffer@nuigalway.ie

$U R L:$ http://schmidt.nuigalway.ie/ goetz

FAkultät Für Mathematik, Ruhr-Universität Bochum, D-44780 Bochum, Germany

E-mail address: gerhard.roehrle@rub.de

URL: http://www.ruhr-uni-bochum.de/ffm/Lehrstuehle/Lehrstuhl-VI 